\title{
An injectable, click-crosslinked, cytomodulin-modified hyaluronic acid hydrogel for cartilage tissue engineering
}

Seung Hun Park', Ji Young Seo', Joon Yeong Park', Yun Bae Ji', Kyungsook Kim', Hak Soo Choi $\mathbb{D}^{2}$, Sangdun Choi', Jae Ho Kim', Byoung Hyun Min ${ }^{1}$ and Moon Suk Kim

\begin{abstract}
This is the first report, to our knowledge, of the preparation of an injectable in situ-forming click-crosslinked hyaluronic acid (CX-HA) hydrogel (CX-HA-CM) containing chemical immobilized cytomodulin-2 (CM), a chondrogenic differentiation factor, and on the utility of human periodontal ligament stem cells (hPLSCs) as a cell source for cartilage tissue engineering. hPLSCs served here as a stem cell source tolerant to ex vivo manipulation. CM induced in vitro chondrogenic differentiation of hPLSCs comparable to induction with traditional TGF- $\beta$. CX-HA was prepared via a click-reaction between tetrazine-modified HA and transcyclooctene-modified HA. CX-HA displayed significantly more features of a stiff hydrogel than HA. CX-HA had a three-dimensional porous interconnected structure, absorbed a large volume of biological medium, and showed excellent biocompatibility. In contrast to HA, the Cx-HA hydrogel persisted in vitro and in vivo for an extended period, as evidenced by in vivo near-infrared fluorescence imaging. CM covalently linked to CX-HA (CX-HA-CM) remained inside Cx-HA for a prolonged period compared with CM physically loaded onto $\mathrm{Cx}-\mathrm{HA}[\mathrm{CX}-\mathrm{HA}(+\mathrm{CM})]$. Cx-HA-CM also caused better chondrogenic differentiation of hPLSCs, as evidenced by Alcian blue and Safranin $\mathrm{O}$ staining, and greater increases in the expression of type II collagen, glycosaminoglycan content and SOX9, aggrecan, and type 2 al collagen mRNA levels. Thus, compared to Cx-HA (+CM), the hPLSC-loaded Cx-HACM hydrogel induced greater chondrogenic differentiation of hPLSCS via CM that was retained in the hydrogel for a much longer period of time.
\end{abstract}

\section{Introduction}

Damaged articular cartilage has limited spontaneous healing potential, and failure to repair such cartilage often leads to osteoarthritis, pain, and malfunction of the affected joint ${ }^{1-3}$. Although many approved therapeutic procedures for restoring the structure of damaged articular cartilage are available, including microfracture, cell implantation, and tissue grafts, these procedures often do not produce robust and well-repaired articular cartilage $^{4-6}$.

\footnotetext{
Correspondence: Moon Suk Kim (moonskim@ajou.ac.kr)

'Department of Molecular Science and Technology, Ajou University, Suwon 16499, Korea

${ }^{2}$ Gordon Center for Medical Imaging, Massachusetts General Hospital, Harvard Medical School, Charlestown, MA 02129, USA
}

Cartilage tissue engineering is considered a promising modality for effective repair of damaged cartilage tissue ${ }^{7,8}$. The procedure most often used in cartilage tissue engineering involves a suitable combination of seeded cells, a biocompatible scaffold, and biological factors that support cartilage formation. The success of cartilage tissue regeneration depends on the individual and/or combined characteristics of the cells, the biological factors, and the scaffold $^{9}$. In this work, we attempted to design a suitable combination of cells and biological factors and a suitable scaffold for cartilage tissue engineering.

First, we chose human periodontal ligament stem cells (hPLSCs) as a cell source for the cartilage tissue engineering in this work because hPLSCs can easily be harvested in large quantities from teeth obtained during

\section{(c) The Author(s) 2019}

(c) (i) Open Access This article is licensed under a Creative Commons Attribution 4.0 International License, which permits use, sharing, adaptation, distribution and reproduction c. in any medium or format, as long as you give appropriate credit to the original author(s) and the source, provide a link to the Creative Commons license, and indicate if changes were made. The images or other third party material in this article are included in the article's Creative Commons license, unless indicated otherwise in a credit line to the material. If material is not included in the article's Creative Commons license and your intended use is not permitted by statutory regulation or exceeds the permitted use, you will need to obtain permission directly from the copyright holder. To view a copy of this license, visit http://creativecommons.org/licenses/by/4.0/. 
dental repair or surgical procedures. Additionally, hPLSCs have been proposed as a self-renewing and multipotent cell source; they have a high proliferation rate during ex vivo expansion and can be cryopreserved for long periods. Furthermore, hPLSCs are capable of differentiation into a chondrogenic lineage and also possess other differentiation potential; this property makes hPLSCs attractive for studies of cartilage tissue engineering ${ }^{10-12}$.

Next, we chose a hyaluronic acid (HA)-based hydrogel for cartilage tissue engineering in this work because HA is a naturally occurring biopolymer that is present in almost all mammalian tissues; due to its biocompatibility, biodegradability, and nontoxicity, it has been widely utilized in a variety of medical treatments ${ }^{13}$. In cartilage tissue regeneration, HA has been employed in various scaffold formats such as cream, film, and foam ${ }^{14-18}$. Some research groups have reported that HA scaffolds stimulate in vitro chondrogenesis of mesenchymal stromal cells (MSCs) and that constructs that are in vitro tissueengineered via chondrogenic differentiation of MSCs in HA scaffolds have properties similar to those of native cartilage $^{19,20}$.

Injectable in situ-forming hydrogels have been widely used as scaffolds for cartilage tissue engineering ${ }^{21-25}$. Injectable in situ-forming hydrogel systems easily and quantitatively incorporate various cells and biological factors after simple mixing at room temperature and can be injected as a liquid to easily form a hydrogel in situ.

Several natural materials, including HA, cellulose, and collagen, have been proposed as injectable in situ-forming hydrogels for cartilage tissue engineering ${ }^{25}$. An injectable HA hydrogel can temporarily maintain its elastic and viscose integrity as a scaffold under physiological conditions. HA hydrogels show good capacity for water absorption and high swelling ability through water absorption. Consequently, the use of an injectable HA hydrogel as a scaffold can create a suitable three-dimensional environment for faster growth of loaded cells by allowing the diffusion of various biological nutrients. Furthermore, the injectable HA hydrogel can itself enhance cartilage-specific synthesis of extracellular matrix by stem cells, as reported elsewhere $^{26,27}$. Thus, injectable in situ-forming HA hydrogels appear promising for use in cartilage tissue engineering. However, once injected, HA hydrogels gradually and completely disappear under physiological conditions due to their short residence time.

It was recently shown that the biorthogonal Diels-Alder click reaction between tetrazine (Tet) and transcyclooctene (TCO) can proceed rapidly under physiological conditions even in the absence of an external catalyst ${ }^{28-32}$. To our knowledge, there are very few published studies on the use of injectable click-cross-linked HA hydrogels in cartilage tissue engineering of hPLSCs. In this work, we designed Tet-modified HA (HA-Tet) and TCO-modified
HA (HA-TCO) and used them in situ to form a clickcrosslinked HA (Cx-HA) hydrogel with prolonged residence time.

Transforming growth factor beta (TGF- $\beta$ ) is an important regulator of crucial cellular processes involved in early embryonic development, cell growth, differentiation, motility, and apoptosis ${ }^{33}$. In addition, TGF- $\beta$ controls the chondrogenic differentiation of MSCs. Thus, TGF- $\beta$ has been added as a key component to nearly all chondrogenic media; however, it requires careful storage to prevent denaturation and is quite costly due to expensive manufacturing steps.

If bioactive short peptides that mimic the action of fulllength TGF- $\beta$ can be designed, they would be attractive alternatives to full-length TGF- $\beta$ owing to their greater stability on storage and cost-effectiveness. For these reasons, the development of short bioactive peptides that mimic the effects of TGF- $\beta$ has recently gained importance. Cytomodulin-2 (CM) is one such TGF$\beta$-mimicking short peptide ${ }^{34-37}$. Although the exact mechanism through which CM mimics TGF- $\beta$ is not known, it has been reported that the $\mathrm{CM}$ peptide acts via a mechanism similar to that of TGF- $\beta$ in which it interacts with cell surface TGF- $\beta$ receptors through the stable and bend structure of the $\mathrm{CM}$ peptide $(-\mathrm{Val}-\mathrm{Ala}-)^{37}$. As a consequence, the TGF- $\beta$-mimicking CM peptide plays a role similar to that of TGF- $\beta$ and enhances the expression of collagen I and pro-collagen I in wound healing. Additionally, the CM peptide has the advantages of longer shelf life and lower cost. Furthermore, one research group has reported that, like TGF- $\beta$, the CM peptide can effectively induce the chondrogenic differentiation of human MSCs (hMSCs) ${ }^{35}$. For these reasons, we chose the $\mathrm{CM}$ peptide in this work as a biological factor for cartilage tissue engineering based on hPLSCs.

For the purpose of cartilage tissue engineering via hPLSCs, the $\mathrm{CM}$ peptide can be physically loaded onto a $\mathrm{Cx}-\mathrm{HA}$ hydrogel by simple mixing. However, the physically CMloaded $\mathrm{Cx}-\mathrm{HA}$ [Cx-HA $(+\mathrm{CM})]$ does not provide an efficacious and stable source of the peptide for the required period of time due to the rapid release of the peptide from the Cx-HA hydrogel. Chemical immobilization of the CM peptide on the Cx-HA hydrogel instead of physical loading is likely to prolong the residence time of $\mathrm{CM}$. Thus, we hypothesized that $\mathrm{Cx}-\mathrm{HA}$ hydrogel $(\mathrm{Cx}-\mathrm{HA}-\mathrm{CM})$ that was chemically modified with CM would enhance the chondrogenic differentiation of hPLSCs due to the resulting prolonged retention of $\mathrm{CM}$ inside the $\mathrm{Cx}-\mathrm{HA}$ hydrogel (Fig. 1). To our knowledge, no published studies have described the use of in situ click-crosslinked Cx-HA-CM for the purpose of cartilage tissue engineering using hPLSCs.

The objectives of this study were to answer the following questions: (1) Can CM induce chondrogenic differentiation of hPLSCs, which are a cell source for 


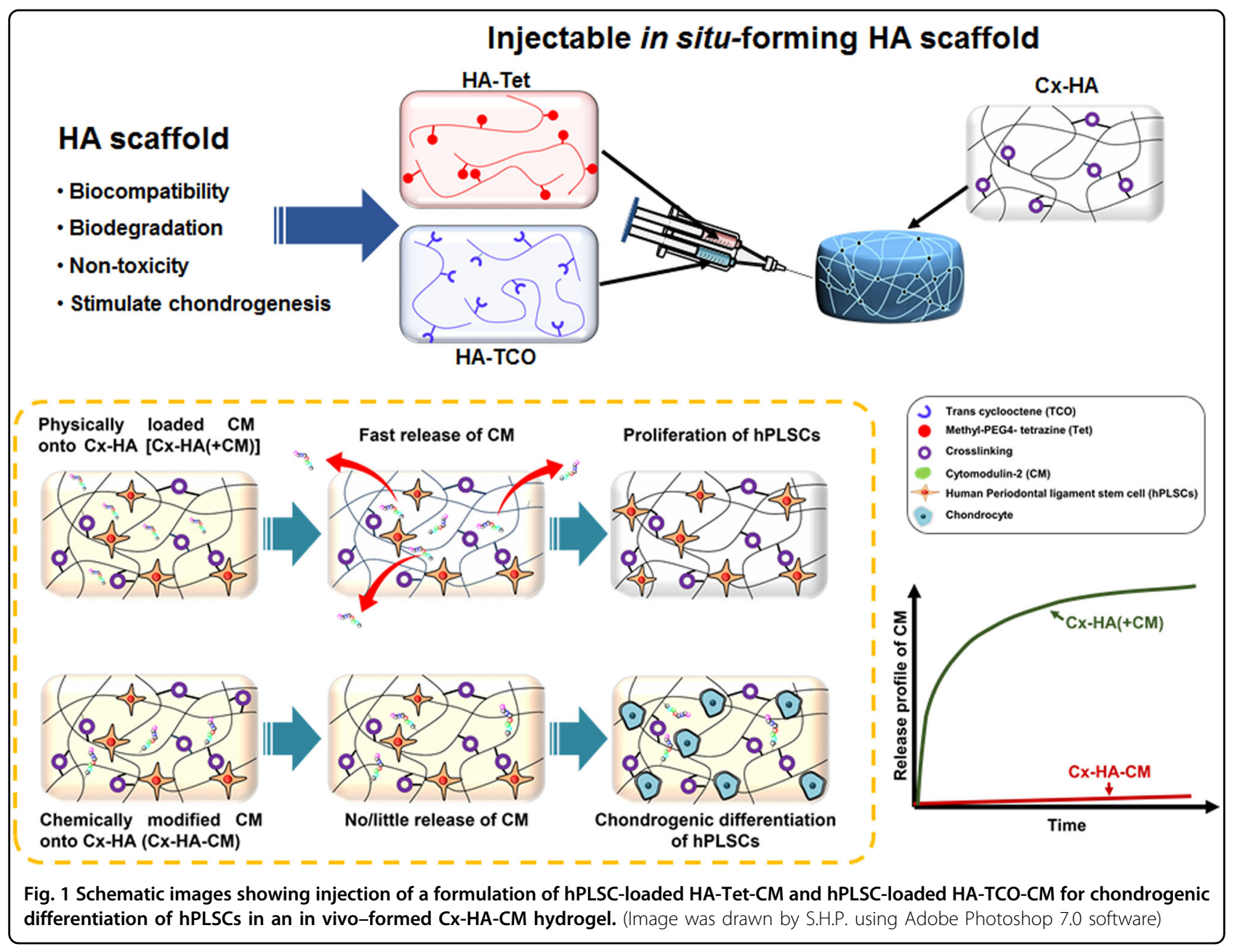

cartilage tissue engineering? (2) Can an injectable clickcrosslinkable-Cx-HA-CM-based hydrogel formulation be introduced into the body to produce a scaffold for hPLSCs in a minimally invasive manner? (3) Can Cx-HA-CM synergistically induce chondrogenic differentiation of hPLSCs more effectively than Cx-HA $(+\mathrm{CM})$ ? Such an injectable Cx-HA-CM-based hydrogel formulation holds promise for satisfying the unmet need for (pre)clinical repair of damaged articular cartilage using safe and inexpensive bioactive short $\mathrm{CM}$ peptides as well as for enhancing the chondrogenic differentiation of hPLSCs as a resource for cartilage tissue engineering.

\section{Materials and methods}

\section{Materials}

Hyaluronic acid (HA) (1 MDa, Humedix, Anyang, Korea), 4,6-dimethoxy-1,3,5-triazin-2yl-4-methylmorpholinium chloride (DMTMM, Sigma, St. Louis, MO, USA), methyltetrazine-PEG4-amine hydrochloride salt (Click Chemistry Tools, AZ, USA), trans-cyclooctene-amine hydrochloride salt (Click Chemistry Tools, AZ, USA), 1,4- diaminobutane (Sigma), cytomodulin-2 (CM, sequence LIANAK) (Peptron, Daejeon, Korea), fluorescein isothiocyanate-conjugated CM (CM-FI) (Peptron, Daejeon, Korea), bromodeoxyuridine (BrdU, Sigma), BrdU antibody (Dako, Glostrup, Denmark), goat anti-mouse IgG antibody conjugated with Alexa Fluor 594 (Invitrogen, CA, USA), collagen type $2 \alpha$ antibody (Abcam, Cambridge, UK), dimethylmethylene blue (DMMB), glycine, $\mathrm{NaCl}$, acetic acid, and chondroitin sulfate from shark cartilage (all from Sigma), PicoGreen reagent (Invitrogen, Carlsbad, CA, USA), $\lambda$ DNA standard and TRIzol Reagent (Invitrogen, CA, USA), SuperScript ${ }^{\circ}$ III First-Strand Kit (Invitrogen), Power SYBR ${ }^{\circ}$ Green (Applied Biosystems, UK), primers for amplification of SOX9, aggrecan (ACAN), and type $2 \alpha 1$ collagen (COL2A1) (Genotech, Daejeon, Korea) were used as received.

\section{Preparation of hPLSCs for in vitro and in vivo chondrogenic differentiation}

Fresh hPLSCs were prepared according to previously described methods ${ }^{38}$. All of the procedures used in this 
study were approved by the Institutional Review Board for Human Subjects Research and Ethics Committee of Inha Hospital (approval No. IUH IRB 12-150).

For preparation of PKH-labeled hPLSCs, PKH67 labeling was performed according to the protocol provided by the manufacturer (Sigma, MO, USA). Briefly, hPLSCs were detached using trypsin-EDTA (Gibco, NY, USA), and the suspended hPLSCs were centrifuged for $5 \mathrm{~min}$ at $2000 \mathrm{rpm}$ and $4{ }^{\circ} \mathrm{C}$. The supernatant was discarded, and the cell pellet was resuspended in serum-free $\alpha$-minimal essential medium ( $\alpha$-MEM; Gibco, NY, USA) to remove serum proteins. The resuspended cells were centrifuged again, the supernatant was removed, and the cell pellet was resuspended in $1 \mathrm{~mL}$ of Diluent C. PKH67 was diluted to a concentration of $4 \times 10^{-6} \mathrm{M}$ in Diluent C. Next, $1 \mathrm{~mL}$ of the PKH67 solution was added to the cell suspension, and the mixture was incubated for $5 \mathrm{~min}$ in the dark at room temperature. To terminate the PKH67 labeling reaction, $2 \mathrm{~mL}$ of fetal bovine serum (FBS, Gibco, NY, USA) was added to the mixture followed by incubation for $1 \mathrm{~min}$. The PKH67-labeled hPLSCs were centrifuged for $5 \mathrm{~min}$ at $2000 \mathrm{rpm}$ and $4{ }^{\circ} \mathrm{C}$. The supernatant was discarded, and the cell pellet was washed twice with $10 \mathrm{~mL}$ of complete medium to ensure the removal of unbound dye. The PKH67-labeled hPLSCs were then used in in vitro experiments.

For preparation of bromodeoxyuridine (BrdU)-labeled hPLSCs, passage-four hPLSCs were cultured in a $75-\mathrm{cm}^{2}$ tissue culture flask (BD Falcon, CA, USA) in $\alpha$-MEM (Gibco, NY, USA) containing 15\% FBS (Gibco, NY, USA), $2 \mathrm{mmol} / \mathrm{L}$ L-glutamine (Gibco, NY, USA), $100 \mu \mathrm{mol} / \mathrm{L}$ ascorbic-acid-2-phosphate (Sigma, MO, USA), 1\% of a penicillin-streptomycin solution (PS, Gibco, NY, USA), and $100 \mu \mathrm{mol} / \mathrm{L}$ BrdU for $24 \mathrm{~h}$. The hPLSCs were then recovered from the flask by washing it out twice with $10 \mathrm{~mL}$ PBS. The BrdU-labeled hPLSCs were subjected to in vivo experiments.

For in vitro chondrogenic differentiation, passage-five hPLSCs $\left(5 \times 10^{5}\right)$ were seeded in $15-\mathrm{mL}$ conical tubes (BD Falcon, MA, USA) and centrifuged at $2000 \mathrm{rpm}$ and $4{ }^{\circ} \mathrm{C}$ for $5 \mathrm{~min}$ to form a pellet of hPLSCs. The pelleted hPLSCs were incubated for $24 \mathrm{~h}$ at $37^{\circ} \mathrm{C}$ in a humidified atmosphere containing $5 \% \mathrm{CO}_{2}$. Each cell pellet was treated with one of three culture media: $1 \mathrm{~mL}$ of a control medium (Cont) $(\alpha-\mathrm{MEM}+15 \% \mathrm{FBS}+1 \%$ L-glutamine $+1 \%$ penicillin-streptomycin $+0.1 \%$ ascorbic acid), $1 \mathrm{~mL}$ of TGF chondrogenic medium ( $\alpha-M E M+1 \%$ non-essential amino acids $+1 \% \%$ penicillin-streptomycin $+1 \%$ ITS + $100 \mathrm{nM}$ dexamethasone $1.25 \mathrm{mg} / \mathrm{mL}$ BSA $+5.35 \mu \mathrm{g} / \mathrm{mL}$ linoleic acid $+50 \mu \mathrm{g} / \mathrm{mL}$ ascorbic acid $+40 \mu \mathrm{g} / \mathrm{mL}$ L-proline $+10 \mathrm{ng} / \mathrm{mL}$ TGF- $\beta$ ), and $1 \mathrm{~mL}$ of a chondrogenic medium $(\mathrm{CM})(\alpha-\mathrm{MEM}+1 \%$ nonessential amino acids $+1 \%$ penicillin-streptomycin $+1 \%$ ITS $+100 \mathrm{nM}$ dexamethasone $+1.25 \mathrm{mg} / \mathrm{mL} \mathrm{BSA}+5.35 \mu \mathrm{g} / \mathrm{mL}$ linoleic acid $+50 \mu \mathrm{g} / \mathrm{mL}$ ascorbic acid $+40 \mu \mathrm{g} / \mathrm{mL}$ L-proline + $100 \mu \mathrm{M} \mathrm{CM})$. The pelleted hPLSCs were cultured for 4 weeks with replacement of the culture medium every 3 days. To confirm chondrogenic induction, hPLSCs were fixed with $4 \%$ paraformaldehyde after 2 and 4 weeks of cultivation. The fixed hPLSCs were embedded in paraffin, and the paraffin block was cut into 4- $\mu$ m-thick slices. The paraffinized slides were deparaffinized twice with xylene at $70{ }^{\circ} \mathrm{C}$ for $2 \mathrm{~h}$.

For hematoxylin and eosin (H\&E) staining, the deparaffinized slides were hydrated in 100, 95, 70, and $60 \%$ ethanol and washed with deionized water (DW). The slides were then washed in running tap water, stained for 5 min with a hematoxylin solution (Sigma), and washed with DW. The slides were immersed in $0.1 \%$ acidic alcohol for $10 \mathrm{~s}$, washed with DW, and incubated in $1 \%$ aqueous ammonia for $1 \mathrm{~min}$; after washing with DW, the slides were stained for $3 \mathrm{~min}$ with an eosin solution (Sigma). The stained slides were fixed and mounted using mounting medium (Muto Pure Chemicals, Tokyo, Japan).

For Safranin O (SO) staining, the hematoxylin-stained slides described in the previous paragraph were stained with Fast Green solution for $5 \mathrm{~min}$ and washed with 1\% acetic acid for $10 \mathrm{~s}$. The slides were then stained with $0.1 \%$ SO for $5 \mathrm{~min}$ and immersed in $95 \%$ ethanol, $100 \%$ ethanol and xylene for $5 \mathrm{~min}$ each. The stained slides were fixed and mounted using mounting medium.

For immunofluorescent staining of type 2 collagen, the slides were hydrated for $5 \mathrm{~min}$ and treated with citrate buffer (Sigma, St. Louis, MO, USA) at $120-130^{\circ} \mathrm{C}$ for $10 \mathrm{~min}$. The slides were washed with PBS and PBS-T (0.05\% Tween 20 in PBS) and blocked with 5\% bovine serum albumin (BSA; Millipore, Billerica, MA, USA) and $5 \%$ horse serum (HS, Gibco, Auckland, New Zealand) in PBS for $90 \mathrm{~min}$ at $37^{\circ} \mathrm{C}$. The sections were incubated for $16 \mathrm{~h}$ at $4{ }^{\circ} \mathrm{C}$ with an antibody against collagen type 2 alpha 1 (Abcam, Cambridge, UK) diluted in antibody diluent (DaKo, Glostrup, Denmark) (1:500), washed with PBS and PBS-T, and incubated with a secondary antibody (goat anti-mouse IgG conjugated with Alexa Fluor ${ }^{\oplus}$ 594; Invitrogen, USA) (1:200) for $3 \mathrm{~h}$ at room temperature in the dark. The slides were then washed with PBS and PBS-T, counterstained with DAPI in DW (1:1000) for $20 \mathrm{~min}$, and mounted using fluorescence mounting solution (DaKo, Glostrup, Denmark). The slides were visualized using an LSM 710 microscope (Carl Zeiss Microimaging GmbH, Göttingen, Germany) and analyzed using ZEN 2009 software (Carl Zeiss Microimaging GmbH, Göttingen, Germany).

\section{Gene expression during in vitro chondrogenic differentiation of hPLSCs}

RNA was isolated from the pellet of hPLSCs using TRIzol Reagent (Invitrogen, CA, USA). RNA was 
quantified on an ND-1000 spectrophotometer (NanoDrop Technologies, DE, USA) at $260 \mathrm{~nm}$, and the concentration and quality of RNA were confirmed by agarose gel electrophoresis. The extracted RNA samples $(50 \mathrm{ng})$ were reverse-transcribed using the SuperScript III First-Strand Kit (Invitrogen). PCR amplification and real-time fluorescence detection of SOX9 (SOX9), aggrecan (ACAN), and type $2 \alpha 1$ collagen (COL2A1) were performed on a Chromo4 Real-Time PCR System (Bio-Rad, CA, USA) with Power SYBR Green (Applied Biosystems, UK). Expression of the genes SOX9, ACAN, and COL2A1 was normalized to the expression of $\alpha$-tubulin $(A T)$. All samples were assayed in triplicate. Data analysis was conducted using the comparative threshold cycle $\left(C_{t}\right)$ method $\left(2^{-\Delta \Delta C t}\right)$ for relative quantification. The PCR primers (GenoTech, Daejeon, Korea) were as follows: SOX9, 5'-ACACACAGCTCACTCGACCTTG-3', 5'-GG GAATTCTGGTTGGTCCTCT-3'; ACAN, 5'-GAGATG GAGGGTGAGGTC-3', 5'-ACGCTGCCTCGGGCTTC -3'; COL2A1, 5'-GGACTTTTCTCCCCTCTCT-3', 5' - G ACCCGAAGGTCTTACAGGA-3'.

\section{Preparation of tetrazine-modified HA (HA-Tet) and transcyclooctene-modified HA (HA-TCO)}

HA was dispersed in DW at a final concentration of $10 \mathrm{mg} / \mathrm{mL}$. DMTMM (35 mg, $0.13 \mathrm{mmol}$ ) was individually added to the HA solution, and the mixture was stirred for $30 \mathrm{~min}$ (Fig. 1). Transcyclooctene-amine hydrochloride salt $(6.6 \mathrm{mg}, 0.025 \mathrm{mmol})$ and methyltetrazine-PEG4amine hydrochloride salt $(9.1 \mathrm{mg}, 0.025 \mathrm{mmol})$ were individually added to the HA solution $(10 \mathrm{mg} / \mathrm{mL})$; the mixture was then stirred for $24 \mathrm{~h}$ at room temperature to prepare tetrazine-modified-HA (HA-Tet) and transcyclooctene-modified-HA (HA-TCO). The HA-Tet and HA-TCO samples were dialyzed for $72 \mathrm{~h}$ to remove unreacted Tet and TCO. After dialysis, the obtained HATet and HA-TCO were lyophilized in a freeze dryer (FD 8508, Ilshinlab, Daejeon, Korea). The yields of HA-TCO and HA-Tet were $91 \%$ and $90 \%$, respectively. The structures of the obtained compounds were confirmed by ${ }^{1} \mathrm{H}$ NMR spectroscopy. Elemental analysis of the amine groups in HA-Tet (C: $39.4 \%, \mathrm{H}: 5.9 \%, \mathrm{~N}: 4.5 \%$ ) and HATCO (C: $39.5 \%, H: 5.7 \%, \mathrm{~N}: 3.95 \%$ ) showed almost quantitative introduction of Tet and TCO.

\section{Preparation of crosslinked HA (Cx-HA)}

The obtained HA-Tet and HA-TCO were individually dispersed in PBS at a final concentration of $20 \mathrm{mg} / \mathrm{mL}$. The HA-Tet and HA-TCO solutions were separately drawn into the individual compartments of a dual-barrel syringe. The $\mathrm{Cx}-\mathrm{HA}$ was prepared by simultaneous injection of the material in each compartment of the dualbarrel syringe into a vial for subsequent characterization.

\section{Characterization of HA, HA-Tet, HA-TCO, and Cx-HA}

To investigate the structure of the obtained material, the ${ }^{1} \mathrm{H}$ nuclear magnetic resonance (NMR) spectra of $\mathrm{HA}$, HA-Tet, HA-TCO, and Cx-HA were recorded using a Mercury Plus 400 instrument (Varian) with $\mathrm{D}_{2} \mathrm{O}$ in the presence of tetramethylsilane as an internal standard.

The rheological properties of HA, HA-Tet, HA-TCO, $\mathrm{Cx}-\mathrm{HA}$ and $\mathrm{Cx}-\mathrm{HA}$ that had been subjected to swelling in PBS for $52 \mathrm{~h}$ were examined using a rheometer (MCR 102, Anton Paar, Ostfildern, Germany) with a Peltier temperature-controlled bottom plate and a 25.0-mm stainless steel parallel plate measuring system. All measurements were conducted at a gap length of $0.3 \mathrm{~mm}$ at $25^{\circ} \mathrm{C}$. The time sweep was $0-100 \mathrm{~s}$, the oscillating frequency was $1 \mathrm{~Hz}$, and the $\gamma$ amplitude was $2 \%$. The frequency sweep was conducted at an oscillating frequency of $0.1-10 \mathrm{~Hz}$ and a $\gamma$ amplitude of $2 \%$. The strain sweep was performed at $0.1-1000 \%$ oscillating strain and $1 \mathrm{~Hz}$ oscillating frequency. The storage modulus $\left(G^{\prime}\right)$, loss modulus $\left(G^{\prime \prime}\right)$, viscosity $(\eta)$, and phase angle $(\tan \delta)$ were calculated by the instrument's software.

\section{Determination of the swelling properties of $\mathrm{Cx}-\mathrm{HA}$}

Two hundred microliters of the prepared $\mathrm{Cx}-\mathrm{HA}$ and HA (4 mg Cx-HA or HA) were individually added to a 20$\mathrm{mL}$ vial; $10 \mathrm{~mL}$ of PBS was then added, and the mixture was incubated at $37^{\circ} \mathrm{C}$. At predetermined time points, the PBS that had not been absorbed by the Cx-HA was removed from the vial. The remaining PBS on the surface of the vial was removed using clean wipes (Yuhan-Kimberly, Seoul, Korea). The vial was then weighed to obtain the weight of wet Cx-HA. Each sample of Cx-HA hydrogel was then lyophilized in a freeze dryer until the residue reached a constant weight, and the vial was weighed to determine the weight of the dry $\mathrm{Cx}-\mathrm{HA}$.

The swelling ratio was defined as follows:

$$
\begin{aligned}
& \text { Swelling ratio }(\%)=[(\text { weight of wet } C x-H A- \\
& \text { weight of dry } C x-H A) \div(\text { weight of } d r y C x-H A)] \times 100 .
\end{aligned}
$$

Preparation of near-infrared (NIR) fluorescence-labeled HA ZW800-1C was prepared according to previously reported methods ${ }^{39}$. ZW800-1C (10.5 mg, $\left.0.01 \mathrm{mmol}\right)$ was solubilized in $10 \mathrm{~mL}$ of DW. DMTMM $(3.3 \mathrm{mg}$, $0.012 \mathrm{mmol}$ ) was added to the ZW800-1C solution, and the mixture was stirred at room temperature for $30 \mathrm{~min}$. 1,4-diaminobutane $(15.4 \mathrm{mg}, 0.18 \mathrm{mmol})$ in $1 \mathrm{~mL}$ of $\mathrm{DW}$ was added dropwise to the activated ZW800-1C solution, and the solution was then stirred for $24 \mathrm{~h}$. The aminemodified ZW800-1C was dialyzed for 3 days to remove unreacted DMTMM and 1,4-diaminobutane and then lyophilized in a freeze dryer. Amine-modified ZW800-1C 
$(2.86 \mathrm{mg}, \quad 0.0025 \mathrm{mmol})$ and DMTMM $(830 \mu \mathrm{g}$, $0.003 \mathrm{mmol}$ ) were individually added to $5-\mathrm{mL}$ aliquots of $10 \mathrm{mg} / \mathrm{mL}$ HA-Tet or HA-TCO solutions prepared in $\mathrm{DW}$, and the mixtures were stirred at room temperature. The obtained NIR-labeled HA-Tet and HA-TCO solutions were dialyzed for 3 days and then lyophilized in a freeze dryer.

\section{Preparation of CM-modified crosslinked HA (Cx-HA-CM)}

DMTMM $(0.6 \mathrm{mg}, 0.002 \mathrm{mmol})$ was individually added to $5 \mathrm{~mL}$ of HA-Tet $(10 \mathrm{mg} / \mathrm{mL})$ and HA-TCO $(10 \mathrm{mg} /$ $\mathrm{mL}$ ) solutions, and the mixtures were stirred for $1 \mathrm{~h}$. The CM solution $(1.6 \mathrm{mg})$ was individually added to the activated HA-Tet and HA-TCO solutions, and the mixtures were then stirred at room temperature for $24 \mathrm{~h}$. The obtained CM-modified HA-Tet and CM-modified HATCO were dialyzed for 3 days and then lyophilized using a freeze dryer.

For in vitro and in vivo imaging, CM-FI was covalently attached to HA-Tet and HA-TCO prepared according to the above method. The obtained CM-FI-modified HA-Tet and CM-FI-modified HA-TCO were dissolved at concentrations of $10 \mathrm{mg} / \mathrm{mL}$ in PBS. The fluorescence intensity of the solutions of CM-FI-modified HA-Tet and CMFI-modified HA-TCO was analyzed on an FP-8200 spectrofluorometer (JASCO, MD, USA), and the results were used to determine the amount of CM-FI that was chemically immobilized on the HA-Tet and HA-TCO. The fluorescence intensity was measured at an excitation wavelength of $495 \mathrm{~nm}$ and an emission wavelength of $516 \mathrm{~nm}$.

\section{In vitro CM-FI release from CM-FI-loaded Cx-HA [Cx-HA $(+\mathrm{CM}-\mathrm{FI})]$ and $\mathrm{CM}-\mathrm{FI}-$ modified CX-HA [CX-HA-CM-FI]}

Each solution of HA-Tet (+CM-FI) and HA-TCO (+CM-FI), or CM-FI-modified HA-Tet and CMFI-modified HA-TCO was individually prepared. Aliquots of the solutions $(200 \mu \mathrm{L})$ were individually added to $20-\mathrm{mL}$ vials and mixed to prepare $\mathrm{CM}-\mathrm{FI}-$ loaded $\mathrm{Cx}-\mathrm{HA}$ [from HA-Tet (+CM-FI)] and HA-TCO (+CM-FI) and CM-FI-modified Cx-HA [from CM-FI-modified HA-Tet and CM-FI-modified HA-TCO]. The Cx-HA (+CM-FI) and $\mathrm{Cx}-\mathrm{HA}-\mathrm{CM}-\mathrm{FI}$ hydrogels contained the same amount of CM-FI $(0.62 \mathrm{mg} / \mathrm{mL}, 0.6 \mathrm{mM})$. Next, $5 \mathrm{~mL}$ of fresh PBS was added to the vials containing $\mathrm{Cx}-\mathrm{HA}(+\mathrm{CM}-\mathrm{FI})$, and the Cx-HA-CM-FI hydrogel was shaken at $100 \mathrm{rpm}$ in an incubator at $37^{\circ} \mathrm{C}$ for 28 days. At the end of this period, $2 \mathrm{~mL}$ of PBS was removed from each vial and replaced with the same volume of fresh PBS. The amount of released CM-FI was measured using an FP-8200 spectrofluorometer. The fluorescence intensity was measured at an excitation wavelength of $495 \mathrm{~nm}$ and an emission wavelength of $516 \mathrm{~nm}$. Three independent release experiments were performed on the $\mathrm{Cx}-\mathrm{HA}(+\mathrm{CM}-\mathrm{FI})$ and Cx-HA-CM-FI hydrogels. The amount of CM-FI cumulatively released in vitro was calculated by comparison with standard calibration curves based on known concentrations of CM-FI.

To determine in vitro CM-FI degradation in PBS, CMFI $(100 \mu \mathrm{g})$ was dissolved in $1 \mathrm{~mL}$ of PBS in a vial and shaken at $100 \mathrm{rpm}$ in an incubator at $37^{\circ} \mathrm{C}$ for up to 28 days. At 7, 14, 21, and 28 days, $200 \mu \mathrm{L}$ of the solution was withdrawn from each vial, and $200 \mu \mathrm{L}$ of fresh PBS was immediately added to restore the volume. The amount of CM-FI in PBS was measured using an FP-8200 spectrofluorometer.

\section{In vitro viability of $\mathrm{hPLSC}$ in $\mathrm{Cx}-\mathrm{HA}$}

Each solution of HA-TCO and HA-Tet, HA-Tet $(+\mathrm{CM})$ and HA-TCO $(+\mathrm{CM})$, or CM-HA-Tet and CM-HA-TCO was individually prepared. hPLSCs were added to each of the solutions as follows: $50 \mu \mathrm{L}$ of a suspension of hPLSCs $\left(1 \times 10^{6}\right.$ cells $\left./ \mathrm{mL}\right)$ was individually added to the wells of a 24-well plate and mixed with hPLSC-loaded Cx-HA $\left(1 \times 10^{5}\right.$ cells $)$ [from HA-Tet and HA-TCO], Cx-HA $(+\mathrm{CM})\left(1 \times 10^{5}\right.$ cells $)$ [from HA-Tet $(+\mathrm{CM})$ and HATCO $(+\mathrm{CM})]$, or the CM-Cx-HA hydrogel $\left(10^{5}\right.$ cells $)$ [from CM-modified HA-Tet and CM-modified HA-TCO]. The $\mathrm{Cx}-\mathrm{HA}(+\mathrm{CM})$ and $\mathrm{CM}-\mathrm{Cx}-\mathrm{HA}$ hydrogels contained the same amounts of $C M(0.4 \mathrm{mg} / \mathrm{mL}, 0.6 \mathrm{mM})$. The concentration of $\mathrm{Cx}-\mathrm{HA}, 20 \mathrm{mg} / \mathrm{mL}$, was the same as well.

The hPLSC-loaded Cx-HA, Cx-HA $(+\mathrm{CM})$, and CxHA-CM hydrogels were incubated for 7 days at $37{ }^{\circ} \mathrm{C}$ and $5 \% \mathrm{CO}_{2}$. For comparison, hPLSC-loaded $\mathrm{Cx}-\mathrm{HA}$ was added to the wells of a 24-well plate by passage through a 26-gauge needle attached to a syringe. All hydrogels were cultivated with replacement of the culture medium every 3 days. 3-(4,5-dimethylthiazol-2-yl)-2,5-diphenyltetrazolium bromide (MTT) assays were performed at 1, 4, and 7 days. For the MTT assay, $100 \mu \mathrm{L}$ of a PBS solution of the MTT substrate (Roche, Basel, Switzerland) was added to each well. After incubation at $37^{\circ} \mathrm{C}$ for $4 \mathrm{~h}$, the resulting violet formazan precipitate was solubilized by the addition of $500 \mu \mathrm{L}$ of DMSO and shaking for $30 \mathrm{~min}$. An aliquot $(100 \mu \mathrm{L})$ of solution from each well was transferred to a 96-well plate. The solutions were then read on an ELISA plate reader (EL808 Ultra microplate reader, Bio-Tek, Winooski, VT, USA). The optical density at $590 \mathrm{~nm}$ of the solution in each well was determined.

PKH-labeled hPLSC-loaded Cx-HA, Cx-HA (+CM), and $\mathrm{Cx}-\mathrm{HA}-\mathrm{CM}$ hydrogels were individually prepared with the same concentrations of PKH-labeled hPLSCs, $\mathrm{CM}$, and $\mathrm{Cx}-\mathrm{HA}$ according to the procedure described in the foregoing subsection. The PKH-labeled hPLSCs on $\mathrm{Cx}-\mathrm{HA}, \mathrm{Cx}-\mathrm{HA}(+\mathrm{CM})$, and $\mathrm{Cx}-\mathrm{HA}-\mathrm{CM}$ hydrogels were examined at 1,7 , and 14 days using an Olympus IX51 inverted fluorescence microscope (Olympus, Tokyo, Japan) and analyzed using Motic Images Advanced 3.2 software (Motic Co., Ltd., Hong Kong, China). 
In vivo imaging of CM-Fl-loaded $\mathrm{Cx}-\mathrm{HA}$ [Cx-HA (+CM-FI)] and $\mathrm{CM}-\mathrm{Fl}$-modified $\mathrm{Cx}-\mathrm{HA}$ [Cx-HA-CM-FI]

Solutions $(200 \mu \mathrm{L} ; 4 \mathrm{mg} \mathrm{Cx}-\mathrm{HA})$ of [HA-Tet $(+\mathrm{CM}-\mathrm{FI})$ and HA-TCO (+CM-FI)] and [HA-Tet-CM-FI and HATCO-CM-FI] with CM-FI $(124 \mu \mathrm{g})$ were separately drawn into the barrels of a dual-barrel syringe and injected into subcutaneous tissue using a 26-gauge needle.

NIR images were obtained using fluorescence excitation at $460 \mathrm{~nm}$ and emission light filtered through a 500- to 525-nm bandpass filter with an exposure time of $500 \mathrm{~ms}$ and a gain of one using a dichroic cube filter $\left(\mathrm{MgF}_{2}\right.$, fused silica filter). At each time point, NIR images were captured using an imaging instrument (FOBI, NeoScience, Suwon, Korea).

\section{In vivo implantation of $\mathrm{hPLSC}$-loaded $\mathrm{Cx}-\mathrm{HA}, \mathrm{Cx}-\mathrm{HA}(+\mathrm{CM})$ and Cx-HA-CM}

The protocol used in the animal experiments in this study was approved by the Institutional Animal Experiment Committee (Approval No. 2017-0015) of Ajou University School of Medicine, and all animals used in this work were treated in accordance with the Guidelines for the Care and Use of Animals for Experimental and Scientific Purposes. Male nude mice (20-22 g, 6 weeks old) were used in accordance with the approved guidelines.

Each solution $(200 \mu \mathrm{L} ; 4 \mathrm{mg} \mathrm{Cx}-\mathrm{HA})$ of [HA-Tet and HA-TCO], [HA-Tet $(+\mathrm{CM})$ and HA-TCO $(+\mathrm{CM})]$, and [HA-Tet-CM and HA-TCO-CM] was prepared using the same amount of CM $(0.4 \mathrm{mg} / \mathrm{mL})$ and the same number of BrdU-labeled hPLSCs $\left(5 \times 10^{6}\right.$ cells $\left./ \mathrm{mL}\right)$. The solutions were then separately drawn into the compartments of a dual-barrel syringe. A 26-gauge needle attached to the dual syringe was used to inject the solutions into the subcutaneous dorsum of nude mice that had been anesthetized with $60 \mu \mathrm{L} / \mathrm{kg}$ Zoletil-Rompun (1:1 solution). The resulting BrdU-labeled hPLSC-loaded Cx-HA, BrdUlabeled hPLSCs-Cx-HA (+CM), and BrdU-labeled hPLSCs-Cx-HA-CM hydrogel implants were allowed to develop and were biopsied after 1, 2, 3, and 4 weeks.

\section{Histological analysis of implants chondrogenically differentiated in vivo}

At $1,2,3$, or 4 weeks after the injection, the experimental mice were individually euthanized. The implants were collected and fixed in 10\% formalin for 7 days. The fixed tissue implants were embedded in paraffin and cut into 4- $\mu \mathrm{m}$ slices. The paraffinized slides were incubated at $80^{\circ} \mathrm{C}$ in an oven for $2 \mathrm{~h}$ to bond the tissue to the glass.

For hematoxylin and eosin (H\&E) staining, the paraffinized slides were deparaffinized with xylene and hydrated in a series of 100, 95, 80, 70, and 60\% ethyl alcohol solutions. The slides were then washed in running tap water, stained with hematoxylin (Muto Pure Chemicals,
Tokyo, Japan) for $3 \mathrm{~min}$, and washed with DW. The hematoxylin-stained slides were sequentially stained with eosin for 2 min and washed with DW. The stained slides were hydrated using 95\% ethyl alcohol, 100\% ethyl alcohol and xylene for $2 \mathrm{~min}$ and were then fixed and mounted in mounting medium (Muto Pure Chemicals, Tokyo, Japan).

For Alcian blue staining, the deparaffinized slides were first washed with DW and then treated with an Alcian blue (Sigma, MO, USA) solution for $30 \mathrm{~min}$. The slides were washed three times with DW. The nuclei of cells in tissues were stained with Nuclear Fast Red (Sigma, MO, USA) and then fixed and mounted using mounting medium.

For SO staining, the deparaffinized slides were washed three times with DW and treated with Mayer's hematoxylin solution for $5 \mathrm{~min}$ followed by washing with DW for $20 \mathrm{~min}$. The stained slides were developed in $0.002 \%$ Fast Green solution (Sigma-Aldrich, St. Louis, MO, USA) for $30 \mathrm{~s}$ and washed with $1 \%$ glacial acetic acid. The slides were then placed in $0.1 \%$ SO solution (Sigma-Aldrich, St. Louis, MO, USA) for $6 \mathrm{~min}$ and fixed using mounting medium.

For immunofluorescent staining for type 2 collagen, the deparaffinized slides were hydrated for $5 \mathrm{~min}$ and then treated with citrate buffer at $120-130^{\circ} \mathrm{C}$ for $10 \mathrm{~min}$. The slides were washed with PBS and PBS-T and blocked with $5 \%$ bovine serum albumin (BSA; Millipore, Billerica, MA, USA) and 5\% horse serum (HS; Gibco, Auckland, New Zealand) in PBS for $90 \mathrm{~min}$ at $37^{\circ} \mathrm{C}$. The slide sections were incubated for $16 \mathrm{~h}$ at $4{ }^{\circ} \mathrm{C}$ with an antibody against collagen type 2 alpha 1 (Abcam, Cambridge, UK) diluted in antibody diluent (DaKo, Glostrup, Denmark) (1:500), washed with PBS and PBS-T, and incubated with a secondary antibody (goat anti-mouse IgG conjugated with Alexa Fluor 594; Invitrogen, USA) (1:200) for $3 \mathrm{~h}$ at room temperature in the dark. The slides were washed with PBS and PBS-T, counterstained with DAPI in DW (1:1000) for $20 \mathrm{~min}$, and mounted using fluorescent mounting solution (DaKo, Glostrup, Denmark).

For macrophage (ED1) staining, the slides were incubated for $10 \mathrm{~min}$ in citrate buffer at $120-130^{\circ} \mathrm{C}$; they were then incubated in PBS for $10 \mathrm{~min}$ and washed with PBS containing 0.05\% Tween 80 (PBST, Sigma, St. Louis, MO, USA). The slides were blocked in PBS containing horse serum (HS; Gibco, Auckland, New Zealand) and 5\% bovine serum albumin (BSA; Roche, Penzberg, Germany) for $90 \mathrm{~min}$ at $37^{\circ} \mathrm{C}$. The slides were incubated at $4{ }^{\circ} \mathrm{C}$ with ED1 (mouse anti-rat CD68 antibody; Serotec, Oxford, UK) in antibody diluent (DAKO, Glostrup, Denmark) (1:1000) for $12-16 \mathrm{~h}$, washed with PBS and PBST, and incubated with a secondary antibody (goat anti-mouse Alexa Fluor 594; Invitrogen, CA, USA) (1:200) for $3 \mathrm{~h}$. The slides were washed again with PBST, counterstained with DAPI, and mounted using fluorescent mounting solution 
(DAKO, Glostrup, Denmark). The slides were visualized using an LSM 710 microscope (Carl Zeiss Microimaging $\mathrm{GmbH}$, Göttingen, Germany) and analyzed using ZEN 2009 software (Carl Zeiss Microimaging GmbH, Göttingen, Germany).

\section{Determination of the GAG and DNA content of in vivo chondrogenically differentiated implants}

The excised hydrogel implants were incubated for $24 \mathrm{~h}$ at $60^{\circ} \mathrm{C}$ in papain solution $(95 \mathrm{mM}$ L-cysteine, $100 \mathrm{mM}$ $\mathrm{Na}_{2} \mathrm{HPO}_{4}, 5 \mathrm{mM}$ EDTA, $125 \mu \mathrm{g} / \mathrm{mL}$ type III papain, $\mathrm{pH}$ 7.5). The resulting solution was centrifuged at $12000 \times g$ for $10 \mathrm{~min}$. For measurement of the GAG content of implants, $20 \mu \mathrm{L}$ of the upper layer obtained after centrifugation was mixed for 5 seconds with $200 \mu \mathrm{L}$ of DMMB solution [16 mg DMMB (Sigma, MO, USA) in $1 \mathrm{~L}$ of water containing $3.04 \mathrm{~g}$ glycine (Sigma, MO, USA), $1.6 \mathrm{~g} \mathrm{NaCl}$ (Sigma, MO, USA), and $95 \mathrm{~mL}$ of $0.1 \mathrm{M}$ acetic acid (Sigma, MO, USA)]. The GAG content was determined using a Cytation 3 (BioTek, VT, USA) at an absorbance of $525 \mathrm{~nm}$. The amount of GAG in implants was calculated through comparison with standard calibration curves prepared using known concentrations of chondroitin sulfate obtained from shark cartilage (Sigma, MO, USA).

For measurement of the DNA content of implants, $100 \mu \mathrm{L}$ of the upper layer obtained by centrifugation was reacted for $5 \mathrm{~min}$ with $100 \mu \mathrm{L}$ of PicoGreen reagent (Invitrogen, Carlsbad, CA, USA) in Tris-EDTA buffer (Sigma, MO, USA) (1:200). DNA content was determined based on the intensity of light emission at $520 \mathrm{~nm}$ after excitation at $480 \mathrm{~nm}$ using a Cytation 3 (BioTek, VT, USA). The amount of DNA in the implants was calculated through comparison with standard calibration curves prepared using known concentrations of a $\lambda$ DNA standard (Invitrogen, Carlsbad, CA, USA). GAG content was normalized to DNA content.

\section{Gene expression in in vivo chondrogenic differentiated implants}

To examine in vivo RNA expression, the hydrogel implants were excised after $1,2,3$, and 4 weeks. In vivo RNA expression was determined using a method similar to the method used in the in vitro experiment described above. All samples were analyzed in triplicate.

\section{Statistical analysis}

Values reflecting in vitro and in vivo gene expression, rheological properties, cytotoxicity, and counts of BrdU-, type 2 collagen-, and ED1-positive cells were obtained from three independent experiments. All data are presented as the mean and standard deviation (SD). To evaluate significance, the results were subjected to oneway analysis of variance (ANOVA) with Bonferroni's multiple-comparison correction in SPSS 12.0 software (SPSS Inc., Chicago, IL, USA).

\section{Results and Discussion}

In vitro chondrogenic differentiation of hPLSCs

To study the stemness and ex vivo expansion of hPLSCs as a cell source for cartilage tissue engineering, hPLSCs isolated from a young woman were allowed to proliferate in culture medium and were expanded up to passage five. The hPLSCs tested positive for MSC markers CD90 $(>99.9 \%)$ and CD166 (>99.8\%) and negative for the hematopoietic stem cell marker CD34 $(<0.01 \%)$. hPLSCs maintained their stem cell characteristics through five passages, indicating their relative tolerance to ex vivo manipulation. Additionally, hPLSCs showed a higher proliferation rate than did hMSCs and were viable after cryopreservation. Thus, hPLSCs are suitable as a source of stem cells for subsequent experiments on chondrogenic differentiation.

Although CM can effectively induce chondrogenic differentiation of $\mathrm{hMSCs}^{34-37}$, to the best of our knowledge the effect of these peptides on hPLSCs has not been investigated. To examine chondrogenic differentiation of hPLSCs under the influence of CM or TGF- $\beta$, hPLSCs were cultured for 4 weeks in culture medium (control) or in CM or TGF- $\beta$ chondrogenic medium (see the experimental section). Because TGF- $\beta$ is a potent growth factor for chondrogenesis, it was chosen as a positive control in the in vitro experiment. The characteristics of chondrogenic differentiation were identified by $\mathrm{H} \& \mathrm{E}, \mathrm{SO}$, and type 2 collagen staining (Fig. 2).

Even after 4 weeks of cultivation, hPLSCs in the control group manifested no chondrogenic characteristics. This result indicates that hPLSCs were unable to differentiate in the absence of CM or TGF- $\beta$ as a chondrogenic factor. In contrast, as shown by $\mathrm{SO}$ and type 2 collagen staining, hPLSCs cultured in CM or TGF- $\beta$ chondrogenic medium showed chondrogenic characteristics after 2 and 4 weeks. These data confirm that $\mathrm{CM}$ induces chondrogenic differentiation of hPLSCs.

To further characterize the chondrogenic differentiation, we performed semiquantitative reverse-transcription PCR to measure the mRNA expression of the genes $S O X 9$, $A C A N$, and COL2A1 (Fig. 2d). $\alpha$-tubulin mRNA expression was used as a reference.

After 2 weeks, we observed little expression of SOX9, $A C A N$, and COL2A1. After 4 weeks, the expression of SOX9, ACAN, and COL2A1 increased in the TGF- $\beta$ and $\mathrm{CM}$ groups but not in the control group. The expression levels of SOX9, ACAN, and COL2A1 in the presence of $\mathrm{CM}$ or TGF- $\beta$ were $\sim 13$ and 15 -fold higher, respectively, than those in the control. At this time point, the SOX9, $A C A N$, and COL2A1 expression levels in the TGF- $\beta$ 


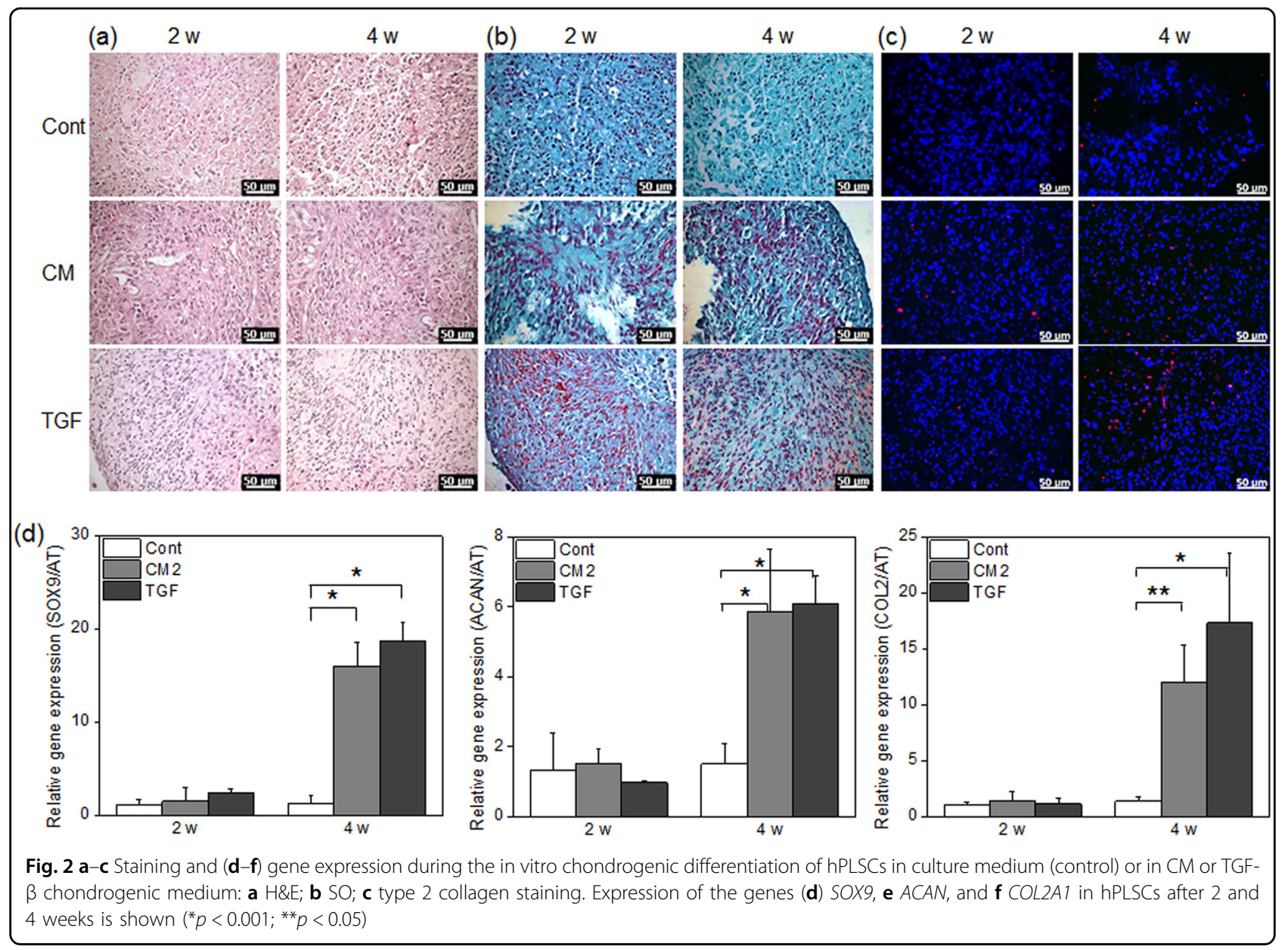

group were only slightly higher than those in the CM group.

These results provide a positive answer to the first key question posed in this study: $\mathrm{CM}$ is almost as effective as TGF- $\beta$ in inducing the chondrogenic differentiation of hPLSCs.

\section{Preparation of an injectable HA hydrogel formulation}

First, the carboxylic group in HA was activated by DMTMM. Then, HA-Tet and HA-TCO were individually prepared by a simple reaction between the activated carboxylic groups in HA and the amine groups in Tet-amine and TCO-amine (Supplementary Fig. S1). The characteristic peaks corresponding to Tet and TCO in HA were observed in the ${ }^{1} \mathrm{H}$-NMR spectra (Supplementary Fig. S2). The yields of HA-Tet and HA-TCO were greater than $90 \%$. Elemental analysis of the amine groups in HATet and HA-TCO showed that there was quantitative introduction of Tet and TCO (Supplementary Table 1).

HA-Tet and HA-TCO were dissolved in PBS. As shown in Supplementary Fig. S2, HA-TCO formed a colorless solution; however, due to the original color of Tet, the
HA-Tet solution was slightly violet in color. Mixing of equal amounts of the HA-Tet and HA-TCO solutions immediately yielded click-crosslinked HA (Cx-HA). The typical time required for $\mathrm{Cx}-\mathrm{HA}$ formation was less than $3 \mathrm{~s}$. The ${ }^{1} \mathrm{H}-\mathrm{NMR}$ spectrum of $\mathrm{Cx}-\mathrm{HA}$ revealed new peaks $f$ and $g$, which appeared after the click reaction between Tet and TCO.

In all of the following experiments, we loaded HA-Tet and HA-TCO in equal amounts into the individual compartments of a dual-barrel syringe; $\mathrm{Cx}$-HA formed immediately via mixing at the injection site. These data provide a positive answer to the second key question posed in our study.

\section{Characterization of HA and Cx-HA hydrogels}

The mechanical properties of HA and Cx-HA were analyzed in rheological experiments that utilized a change in frequency from 0.1 to $10 \mathrm{~Hz}$ (Fig. 3). The strain sweep of the two hydrogels was determined from $0.1 \%$ to $1000 \%$ strain at a frequency of $1 \mathrm{~Hz}$; the limiting strain amplitudes of $\mathrm{HA}$ and $\mathrm{Cx}-\mathrm{HA}$ were found to be $\sim 62.8$ and $99.5 \%$, respectively (Fig. 3a). HA and Cx-HA were stable over the tested 


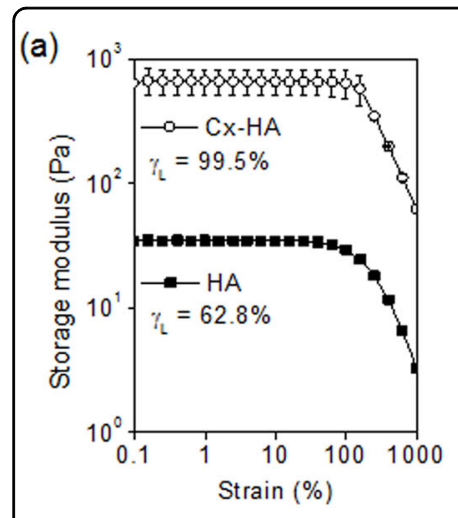

(e)

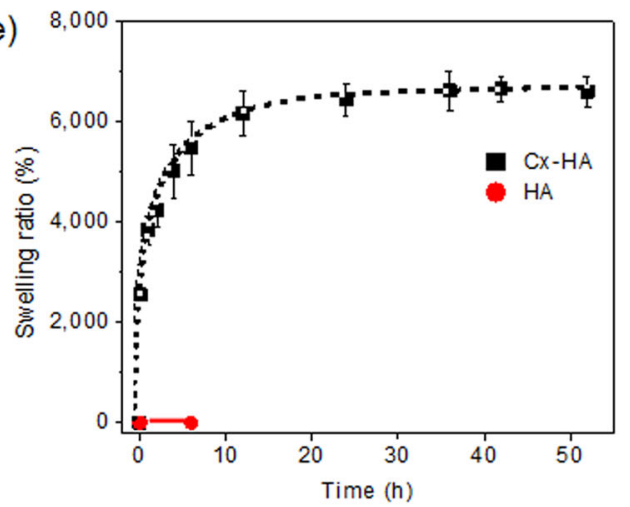

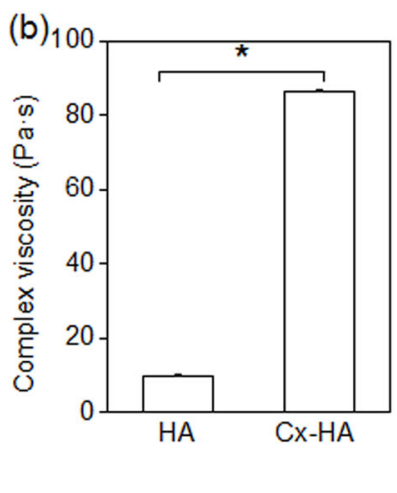

(c)

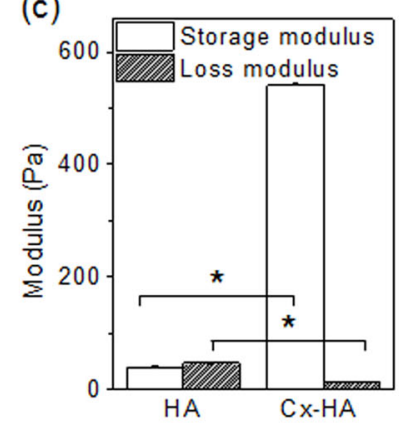

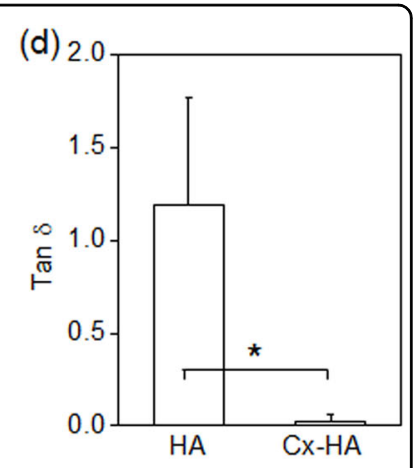

Fig. 3 Rheological characterization of HA and Cx-HA: a the strain sweep test; $\mathbf{b}$ viscosity analysis; $\mathbf{c}$ storage and loss modulus measurements; d phase angle experiment; e swelling ratio versus time; $\mathbf{f}$ imaging of $\mathrm{CX}-\mathrm{HA}$ before and after swelling; $\mathbf{g}$ SEM image of CX-HA ( $\left.{ }^{*} p<0.001\right)$

frequency range of 0.1 to $10 \mathrm{~Hz}$, but $\mathrm{Cx}-\mathrm{HA}$ manifested a higher storage modulus than HA; these data indicate that $\mathrm{Cx}$-HA possesses greater mechanical stiffness than HA.

The complex viscosity of $\mathrm{Cx}$-HA was 10 -fold higher than that of HA (Fig. 3b). The storage $\left(G^{\prime}\right)$ and loss $\left(G^{\prime \prime}\right)$ moduli of $\mathrm{HA}$ and $\mathrm{Cx}-\mathrm{HA}$ were measured at $37^{\circ} \mathrm{C}$ (Fig. $3 \mathrm{c})$. The storage modulus of $\mathrm{Cx}-\mathrm{HA}$ was found to be higher than that of $\mathrm{HA}$, and the loss modulus of $\mathrm{Cx}-\mathrm{HA}$ was lower than that of HA. The difference between the storage and loss values of $\mathrm{Cx}-\mathrm{HA}$ was larger than the difference between the storage and loss values of the HA hydrogel. This result suggests that $\mathrm{Cx}-\mathrm{HA}$ possesses good elastic characteristics.

Next, the elasticity levels of these materials were compared by determining the phase angles $(\tan \delta)$ of $\mathrm{HA}$ and Cx-HA (Fig. 3d). The tan $\delta$ value of HA was 1.2, indicative of predominantly viscous behavior. The $\tan \delta$ value of $\mathrm{Cx}$ HA was found to be 0.02 , suggesting that the hydrogel strength parameters of $\mathrm{Cx}-\mathrm{HA}$ are better, i.e., that $\mathrm{Cx}-\mathrm{HA}$ is significantly stiffer. Collectively, these results indicate that in many respects $\mathrm{Cx}$-HA forms a significantly stiffer hydrogel than HA.

Next, the swelling ratios of HA and $\mathrm{Cx}-\mathrm{HA}$ were compared. HA was completely solubilized in PBS within $15 \mathrm{~min}$ (Fig. 3e). In contrast, Cx-HA maintained the shape of HA but absorbed $2500 \%$ of its volume of PBS in 10 min, 3900\% of its volume in $1 \mathrm{~h}$, and $6600 \%$ of its volume over a period of $24 \mathrm{~h}$ (Fig. 3f). This result indicates that $\mathrm{Cx}-\mathrm{HA}$ can rapidly absorb a large volume of biological medium. Furthermore, in SEM, the Cx-HA hydrogel was found to have a porous interconnected three-dimensional structure (Fig. 3g).

Next, the mechanical properties of $\mathrm{Cx}-\mathrm{HA}$ before and after swelling were analyzed (Supplementary Fig. S3). After swelling, $\mathrm{Cx}-\mathrm{HA}$ exhibited $70 \%$ of the complex viscosity exhibited by $\mathrm{Cx}-\mathrm{HA}$ before swelling. The storage and loss moduli of HA and Cx-HA were measured. The storage and loss moduli of $\mathrm{Cx}$-HA after swelling were found to be slightly lower than those observed before swelling. The difference in the storage and loss moduli of $\mathrm{Cx}$-HA after swelling was still large. This result shows that the elastic characteristics of $\mathrm{Cx}-\mathrm{HA}$ are maintained after swelling.

Collectively, these results support the basic assumption of this study that by permitting the diffusion of biological nutrients, the Cx-HA hydrogel performs well as a threedimensional scaffold for growth, proliferation, and differentiation of the loaded hPLSCs.

\section{In vivo persistence of $\mathrm{HA}$ and $\mathrm{Cx}-\mathrm{HA}$}

The in vivo persistence of the hydrogels was studied because it is important to their function as a durable 


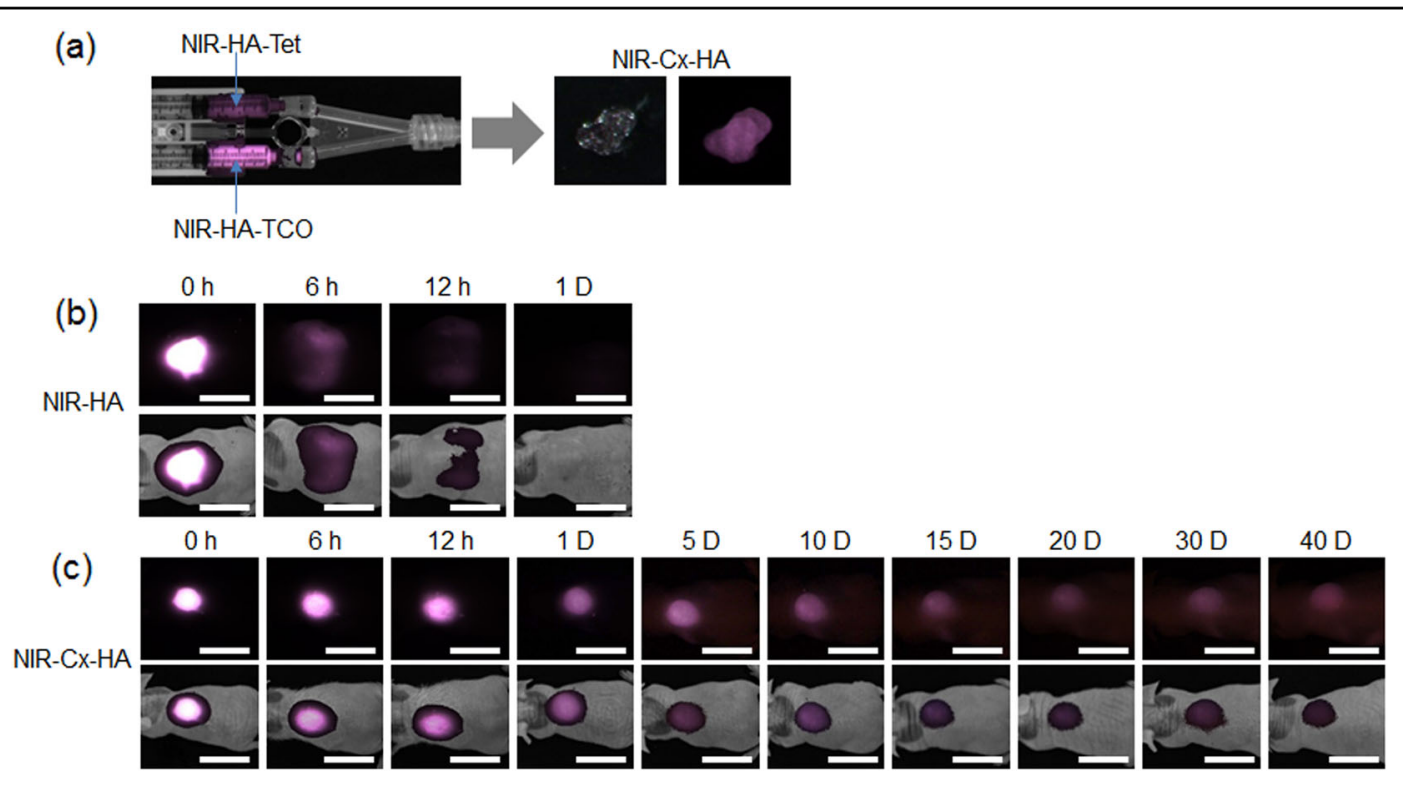

Fig. 4 a Light microscopy and near-infrared (NIR) images of NIR-CX-HA and of HA-Tet and HA-TCO formulations in a dual-barrel syringe. NIR images after injection of (b) NIR-HA and (c) NIR-Cx-HA are shown (scale bars $=20 \mathrm{~mm}$ )

scaffold for hPLSC-based cartilage tissue engineering. NIR images of NIR fluorescence-labeled HA or Cx-HA were captured to assess the in vivo persistence of $\mathrm{HA}$ and $\mathrm{Cx}$ HA hydrogels.

First, the prepared NIR-HA-Tet and NIR-HA-TCO solutions were loaded into the compartments of a dualbarrel syringe, as illustrated in Fig. 4a. Mixing of the NIRHA-Tet and NIR-HA-TCO solutions resulted in the formation of NIR-Cx-HA. The prepared NIR-HA or the NIR-HA-Tet and NIR-HA-TCO solutions were individually subcutaneously injected into nude mice. Immediately after the injection, an NIR-HA or NIR-Cx-HA hydrogel formed at the injection site.

NIR fluorescent images of NIR-HA could be captured until $12 \mathrm{~h}$ after injection, but no signals were observed at 1 day after injection (Fig. 4b). In contrast, NIR fluorescent images of the Cx-HA hydrogel could be successfully captured in vivo for at least 40 days (Fig. 4c). This result indicates that the Cx-HA hydrogel persisted in vivo for a much longer period than the HA hydrogel.

Collectively, our results corroborate the basic assumption of this study, that is, that for a predefined experimental period, the Cx-HA hydrogel is suitable as a scaffold of hPLSCs.

In vitro and in vivo release of $\mathrm{CM}-\mathrm{FI}$ from physically $\mathrm{CM}$ FI-loaded Cx-HA [Cx-HA ( + CM-FI)] and from CM-

FI-modified Cx-HA [Cx-HA-CM-FI]

We compared the persistence of $\mathrm{CM}$ inside $\mathrm{Cx}-\mathrm{HA}$ after the physical and chemical loading of $\mathrm{CM}$ into the hydrogel. First, to monitor the in vitro degradation of
CM-FI, a solution of free CM-FI was incubated at $37^{\circ} \mathrm{C}$ for 28 days, and its fluorescence intensity was then determined. The fluorescence intensity of CM-FI did not change markedly over the 28-day period, indicating that little or no degradation occurred for at least 28 days under our in vitro experimental conditions (Supplementary Fig. S4).

Next, the in vitro cumulative release of CM-FI from CxHA (+CM-FI) and Cx-HA-CM-FI was studied (Fig. 5a, b). From Cx-HA (+CM-FI), CM-FI showed $44 \%$ release at $5 \mathrm{~min}, 83 \%$ release at $12 \mathrm{~h}$, and $92 \%$ release at 1 day, indicating an initial burst of release at 1 day. This shows that physically loaded CM-FI is almost completely liberated from $\mathrm{Cx}-\mathrm{HA}$ after only 1 day.

The release of CM-FI from Cx-HA-CM-FI showed the following pattern: $1.3 \%$ of the fluorescence intensity was released after 1 day, 2.4\% was released after 7 days, and 6.3\% was released at 28 days, indicating that, compared with Cx-HA (+CM-FI), only a slow release of CM-FI occurs. This result indicated that chemical attachment of $\mathrm{CM}$ to the $\mathrm{Cx}-\mathrm{HA}$ hydrogel prolonged the in vitro residence period of CM inside the Cx-HA hydrogel.

To examine the in vivo persistence of CM-FI release by real-time imaging, $\mathrm{Cx}-\mathrm{HA}(+\mathrm{CM}-\mathrm{FI})$ and $\mathrm{Cx}-\mathrm{HA}-\mathrm{CM}-\mathrm{FI}$ were injected (separately) into the subcutaneous tissue of mice (Fig. 5c). The injected Cx-HA (+CM-FI) and CxHA-CM-FI emitted green fluorescent signals at the initial time point of $30 \mathrm{~min}$. The fluorescence intensity of $\mathrm{Cx}$ HA (+CM-FI) gradually decreased over a 4-day period and was almost lost by day 4; only negligible fluorescence was detectable on day 10 . In the mouse that received an 

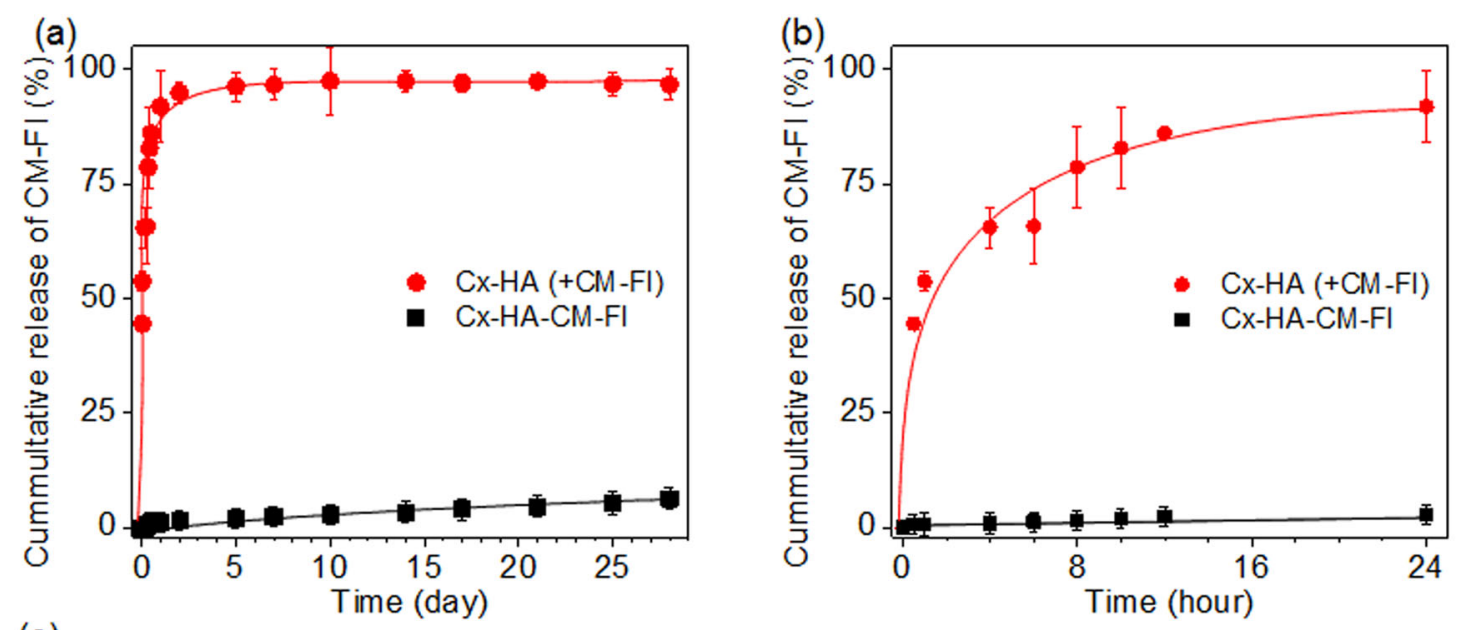

(c)

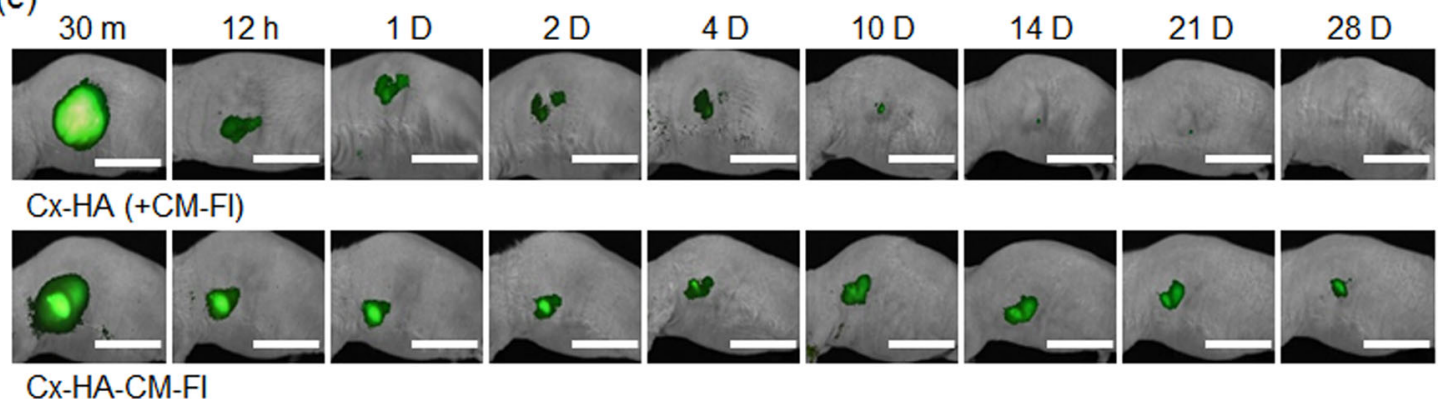

Fig. $\mathbf{5}$ a, $\mathbf{b}$ In vitro and (c) in vivo CM-FI release from CX-HA (+CM-FI) and CX-HA-CM-FI hydrogels (scale bars $=20 \mathrm{~mm})$. Data are presented for (a) a 28-day period and (b) for a shorter time period of $24 \mathrm{~h}$

injection of Cx-HA-CM-FI, fluorescent signals were observed at $30 \mathrm{~min}$, and they persisted for 28 days after the injection.

These results support the basic assumption of this study, namely, that chemical attachment of $\mathrm{CM}$ to $\mathrm{Cx}-\mathrm{HA}$ results in persistent in vitro and in vivo $\mathrm{CM}$ release from the $\mathrm{Cx}$-HA hydrogel for a predefined experimental period.

\section{In vitro and in vivo viability of $\mathrm{hPLSC}$ in $\mathrm{Cx}-\mathrm{HA}, \mathrm{Cx}-\mathrm{HA}$ (+CM), and Cx-HA-CM hydrogels}

Although HA is widely known as a biocompatible biomaterial $^{13}$, we measured the viability of PKH-labeled hPLSCs in vitro. $\mathrm{Cx}-\mathrm{HA}, \mathrm{Cx}-\mathrm{HA}(+\mathrm{CM})$, and $\mathrm{Cx}-\mathrm{HA}-\mathrm{CM}$ containing PKH-labeled hPLSCs were loaded into multiwell plates to evaluate their biocompatibility over a 7-day period (Supplementary Fig. S5). At 1 day, similar numbers of PKH-labeled hPLSCs were observed on Cx-HA, Cx-HA $(+\mathrm{CM})$, and $\mathrm{Cx}-\mathrm{HA}-\mathrm{CM}$ hydrogels. The PKH-labeled hPLSCs proliferated for 7 days in all the Cx-HA-based hydrogels. The number of PKH-labeled hPLSCs on the hydrogels did not differ significantly among the groups. This finding suggests that all of the Cx-HA-based hydrogels are biocompatible with hPLSCs.
Next, the in vitro viability of hPLSCs after passage of the cells through syringe needles during the injection process was examined. There were slight differences in the viability of the cells before and after passage through the needle; however, the hPLSCs showed high viability (80-92\%), comparable to that of hPLSCs before passage through the needle (Supplementary Fig. S6). We confirmed that the injectable click-crosslinked formulation prepared in this work may act as a cell carrier when the cells are forcibly passed through the syringe needles, although the viability of the hPLSCs was slightly affected by the injection method ${ }^{40,41}$.

Next, to examine the in vivo viability of hPLSCs in the injectable hydrogel, injectable $\mathrm{Cx}-\mathrm{HA}, \mathrm{Cx}-\mathrm{HA}(+\mathrm{CM})$, and Cx-HA-CM, together with BrdU-labeled hPLSCs, were injected subcutaneously into nude mice. Immediately after injection, all of the formulations formed $\mathrm{Cx}-\mathrm{HA}$ hydrogel-based scaffolds that maintained their shape over the entire 4-week experimental period; there were no signs of inflammation at the injection site. Thin fibrous capsules containing fibroblasts and blood vessels were visible around the Cx-HA hydrogel (Supplementary Fig. S7). 


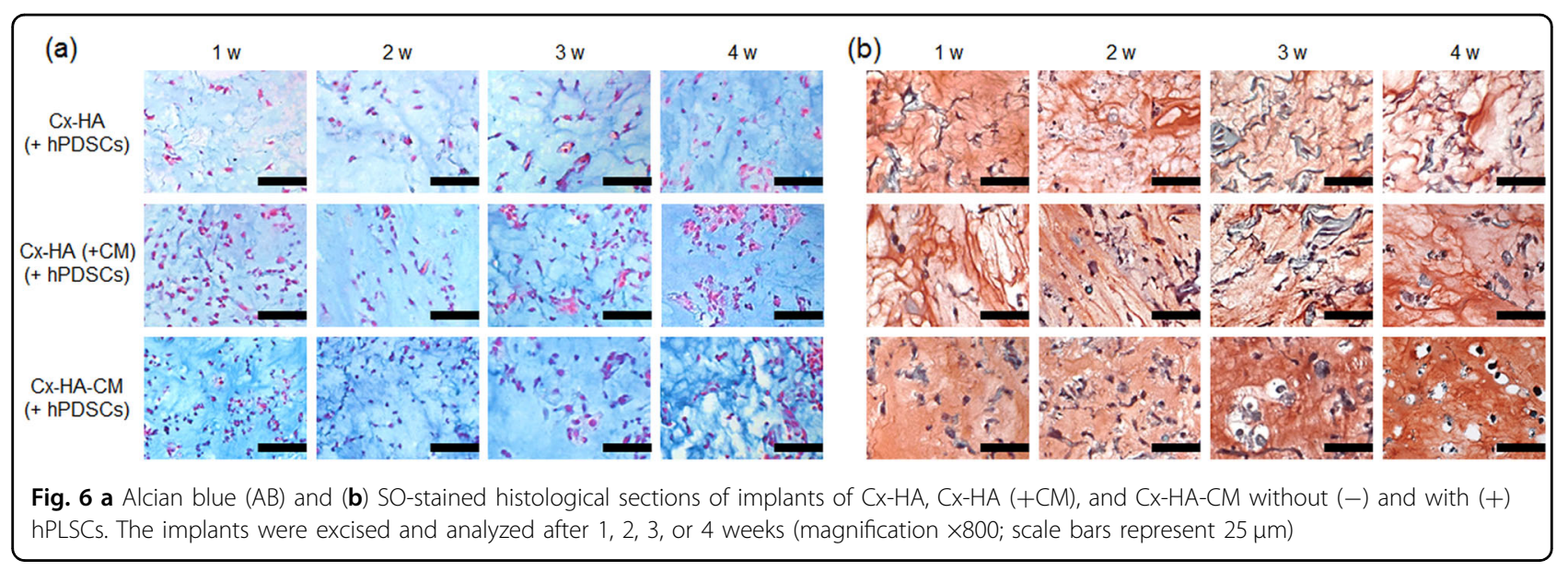

BrdU-labeled hPLSCs (red) and nucleic acid-stained hPLSCs (DAPI, blue) in the Cx-HA hydrogel in vivo were examined by fluorescence microscopy at $1,2,3$, and 4 weeks after injection, and the cells were counted (Supplementary Fig. S8). The intensity of the red signal assignable to BrdU-labeled hPLSCs in the images was similar in samples of the $\mathrm{Cx}-\mathrm{HA}, \mathrm{Cx}-\mathrm{HA}(+\mathrm{CM})$, and $\mathrm{Cx}-$ HA-CM hydrogels. The number of BrdU-labeled hPLSCs did not differ significantly among the groups. These results show that hPLSCs survived well in vivo in the $\mathrm{Cx}$ HA hydrogel for 4 weeks due to the excellent biocompatibility of the Cx-HA hydrogel.

It is well known that macrophage ED1 expression is a unique in vivo indicator of the inflammatory response of host tissues to implants ${ }^{42}$. Accordingly, to examine the inflammatory response of the host tissue to $\mathrm{Cx}-\mathrm{HA}, \mathrm{Cx}-$ HA $(+\mathrm{CM})$, and Cx-HA-CM containing hPLSCs, all of the hydrogel implants were evaluated by immunohistochemical ED1 staining of macrophages (Supplementary Fig. S9). Relative to the number of nuclei stained with DAPI (blue), very few ED1 (red)-stained macrophages were observed among the ED1-postive cells. This result indicates that $\mathrm{Cx}-\mathrm{HA}$ is highly biocompatible.

\section{Histological characteristics of implants chondrogenically differentiated in vivo}

To examine the in vivo chondrogenic differentiation of hPLSCs in Cx-HA-based hydrogels, each injectable formulation (Cx-HA, Cx-HA (+CM), and Cx-HA-CM) was subcutaneously injected with and without hPLSCs into nude mice. At 1, 2, 3 and 4 weeks after the injection, samples of the injected material were stained with H\&E (Supplementary Fig. S10). H\&E-stained histological sections of implants of $\mathrm{Cx}-\mathrm{HA}, \mathrm{Cx}-\mathrm{HA}(+\mathrm{CM})$, and $\mathrm{Cx}-\mathrm{HA}-$ CM without hPLSCs showed little or no tissue organization. In contrast, implants of $\mathrm{Cx}-\mathrm{HA}, \mathrm{Cx}-\mathrm{HA}(+\mathrm{CM})$, and Cx-HA-CM with hPLSCs displayed a more organized tissue-like structure.
The implants of Cx-HA, Cx-HA (+CM), and Cx-HACM with or without hPLSCs were subjected to Alcian blue (AB) and SO staining to confirm the presence of GAG (Fig. 6 and Supplementary Fig. S11). The blue images in group $A B$ and the brown images in group $\mathrm{SO}$ were attributed to the presence of a cartilaginous matrix of GAG. The red images in group $\mathrm{AB}$ and the gray images in group $\mathrm{SO}$ were attributed to the nucleic acids present in the aggregated hPLSCs.

Only slight $A B$ and $S O$ staining was observed in $\mathrm{Cx}-\mathrm{HA}$, Cx-HA $(+\mathrm{CM})$, and Cx-HA-CM implants without hPLSCs, even after 4 weeks. In contrast, an organized cartilaginous matrix and aggregated hPLSCs were found in implants of scaffolds Cx-HA, Cx-HA $(+\mathrm{CM})$, and $\mathrm{Cx}-$ HA-CM containing hPLSCs. The Cx-HA-CM hydrogel with hPLSCs contained the most organized cartilaginous matrix and contained aggregated hPLSCs. Especially after 2 weeks, Cx-HA-CM with hPLSCs showed some rounded morphology that appeared to be due to the presence of chondrocytic lacunae. Chondrocytic lacunae surrounded by a deposition of GAG (brown color) were clearly visible, mostly after 3 and 4 weeks. These results suggest that chemical attachment of $\mathrm{CM}$ to the Cx-HA enhanced the chondrogenic differentiation of hPLSCs to a greater extent than $\mathrm{Cx}-\mathrm{HA}(+\mathrm{CM})$.

Collectively, these results support the main hypothesis that constitutes the third key question of this study; i.e., they show that $\mathrm{CM}$ that remains inside $\mathrm{Cx}$-HA for a long time can synergistically induce the chondrogenic differentiation of hPLSCs for cartilage tissue engineering.

\section{Expression of type II collagen and GAG content of implants chondrogenically differentiated in vivo}

Given that type 2 collagen can be expressed in chondrogenically differentiated implants as the dominant extracellular matrix protein in a cartilaginous matrix ${ }^{43}$, the expression of type 2 collagen was analyzed by immunohistochemical staining of $\mathrm{Cx}-\mathrm{HA}, \mathrm{Cx}-\mathrm{HA}$ 


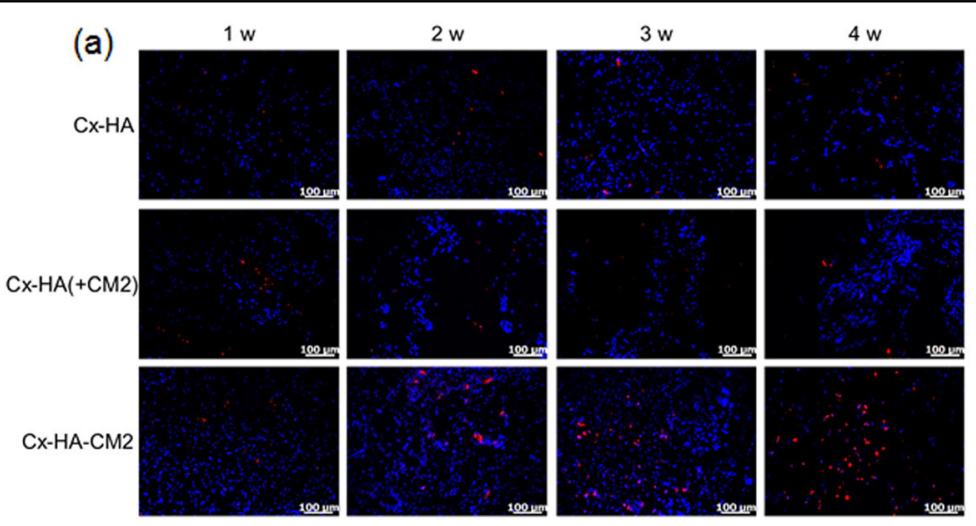

(b)

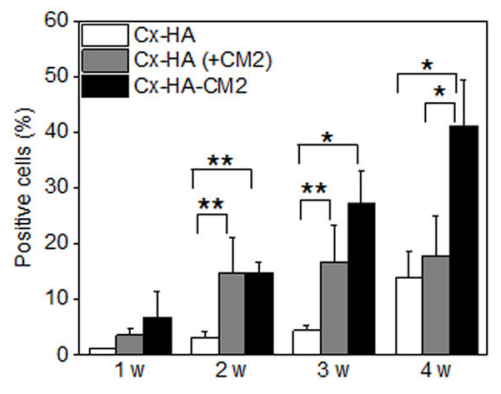

Fig. 7 Histological sections of Cx-HA, Cx-HA ( $+\mathrm{CM})$, and Cx-HA-CM implants after immunofluorescent staining for type 2 collagen. $\mathrm{hPLSCS}$ were removed after 1, 2, 3, or 4 weeks (magnification 200x; scale bars represent $100 \mu \mathrm{m})\left({ }^{*} p<0.001\right.$; ** $p<0.05$ )

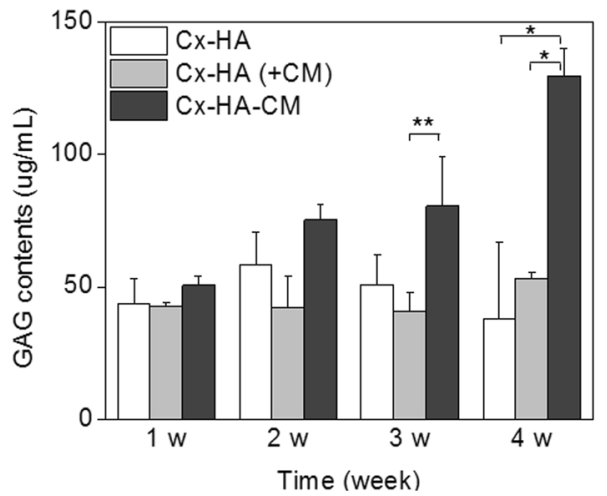

Fig. 8 GAG concentration in $\mathrm{Cx}-\mathrm{HA}, \mathrm{Cx}-\mathrm{HA}(+\mathrm{CM})$, and $\mathrm{Cx}-\mathrm{HA}-\mathrm{CM}$ hydrogels containing hPLSCs. The hydrogel implants were removed after $1,2,3$, or 4 weeks $\left({ }^{*} p<0.001\right.$; $\left.{ }^{* *} p<0.05\right)$

$(+\mathrm{CM})$, and Cx-HA-CM implants containing hPLSCs after 1, 2, 3, or 4 weeks (Fig. 7). The type 2 collagen-positive cells present in the hydrogels were identified and counted.

Cx-HA with hPLSCs showed only weak positive staining (violet color), but greater positive staining for type 2 collagen was observed after 4 weeks, probably due to in vivo chondrogenesis by hPLSCs under the action of the HA scaffold itself ${ }^{26}$. In contrast, positive staining of $\mathrm{Cx}$ HA $(+\mathrm{CM})$ with hPLSCs was barely detectable at 1 week but increased sharply at 2 weeks and then remained almost unchanged for 4 weeks. The reason for this is probably that $\mathrm{CM}$ was released from $\mathrm{Cx}-\mathrm{HA}$ for 10 days, consistent with the results of the experiments with CM-FI described in a previous subsection; thus, $\mathrm{CM}$ was not able to induce the chondrogenic differentiation of hPLSCs during an extended period.

Although Cx-HA-CM with hPLSCs exhibited weak positive staining for type 2 collagen after 1 week, Cx-HA$\mathrm{CM}$ with hPLSCs showed stronger positive staining than either $\mathrm{Cx}-\mathrm{HA}$ or $\mathrm{Cx}-\mathrm{HA}(+\mathrm{CM})$. After 1 week, the positive staining increased gradually and rapidly as a function of post-implantation time. This result suggests that due to the action of the CM that remained inside the $\mathrm{Cx}-\mathrm{HA}$ for an extended period, hPLSCs differentiated into cartilagelike tissues, resulting in the abundant synthesis of type 2 collagen.

GAGs are important components of cartilage, of the synovial fluid that lubricates joints, and of connective tissues and tendons ${ }^{44}$. Consequently, the GAG content of the implants of $\mathrm{Cx}-\mathrm{HA}, \mathrm{Cx}-\mathrm{HA}(+\mathrm{CM})$, and $\mathrm{Cx}-\mathrm{HA}-\mathrm{CM}$ with hPLSCs was quantified after $1,2,3$, and 4 weeks (Fig. 8). At 1 week, all of the implants showed low GAG content, and the GAG content did not differ significantly among the groups. Small amounts of GAGs in the Cx-HA and $\mathrm{Cx}-\mathrm{HA}(+\mathrm{CM})$ hydrogels showed almost no variation in GAG content, although they increased with time postimplantation. In contrast, the GAG content of implants of Cx-HA-CM with hPLSCs was clearly higher than that of the implants in the $\mathrm{Cx}-\mathrm{HA}$ and $\mathrm{Cx}-\mathrm{HA}(+\mathrm{CM})$ with hPLSCs groups. The GAG content of Cx-HA-CM with hPLSCs increased gradually over the 4-week period; in addition, there was a significant difference in GAG content between $\mathrm{Cx}-\mathrm{HA}(+\mathrm{CM})$ and $\mathrm{Cx}-\mathrm{HA}-\mathrm{CM}$ after 3 and 4 weeks $(p<0.05$ and 0.001 , respectively). These data suggest that chondrogenic differentiation of hPLSCs inside the $\mathrm{Cx}$-HA hydrogel induced the formation of a larger amount of GAGs due to the retention of CM in the hydrogel for a long time. The high concentration of GAGs in the Cx-HA$\mathrm{CM}$ implants is in good agreement with the results of $\mathrm{AB}$ and $\mathrm{SO}$ staining described in a preceding subsection.

\section{Gene expression in implants chondrogenically differentiated in vivo}

The formation of cartilage-like tissues in in vivo chondrogenically differentiated implants was examined by semiquantitative PCR analysis of SOX9, ACAN, and 
(a)

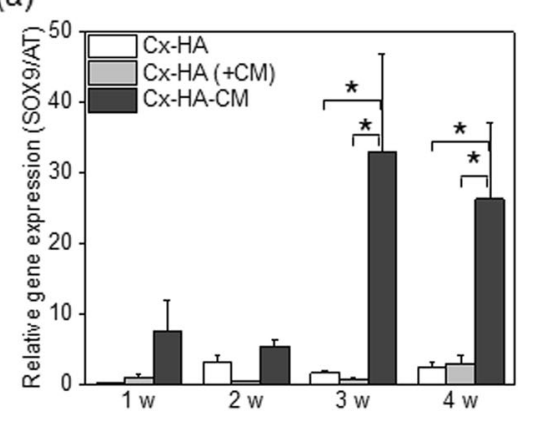

(b)

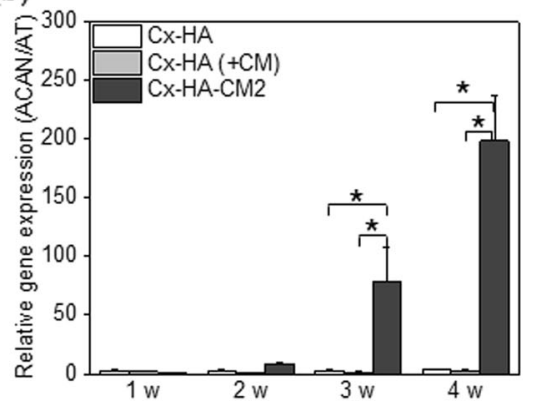

(c)

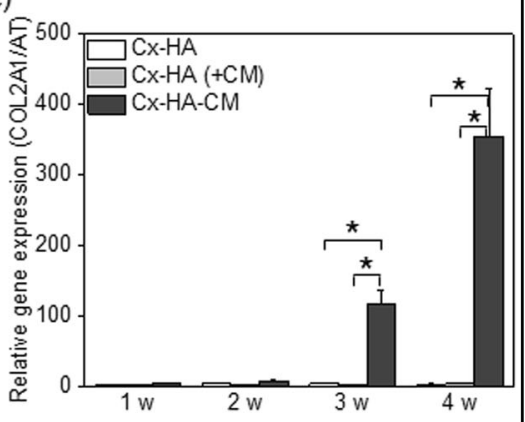

Fig. 9 a SOX9, (b) ACAN, and (c) COL2A1 mRNA expression levels in CX-HA, CX-HA (+CM), and CX-HA-CM implants containing hPLSCs. The implants were excised after $1,2,3$, or 4 weeks $\left({ }^{*} p<0.001\right)$

COL2A1 expression at $1,2,3$, and 4 weeks after in vivo injection (Fig. 9). The in vivo gene expression patterns were almost identical to the in vitro patterns described in a previous subsection. After $1,2,3$, and 4 weeks, the mRNA expression of SOX9, ACAN, and COL2A1 in the $\mathrm{Cx}-\mathrm{HA}-\mathrm{CM}$ hydrogel containing hPLSCs was clearly higher than that in $\mathrm{Cx}-\mathrm{HA}$ and $\mathrm{Cx}-\mathrm{HA}(+\mathrm{CM})$ hydrogels with hPLSCs.

At 3 weeks, the mRNA expression of SOX9, an early marker of the formation of a cartilage-like tissue matrix $^{45,46}$, in the Cx-HA-CM hydrogel with hPLSCs was clearly higher than its expression at 4 weeks. The mRNA levels of $A C A N$ and COL2A1, two markers of the final stage of chondrogenic differentiation ${ }^{42}$, increased gradually as a function of time in the Cx-HA-CM hydrogel containing hPLSCs and showed significantly higher expression after 4 weeks $(p<0.001)$. This result indicates that the chondrogenic differentiation of hPLSCs inside the Cx-HA hydrogel induces the formation of cartilage-like tissues by $\mathrm{CM}$ that is retained within the hydrogel for a long time.

Collectively, these results support a positive answer to our third key question, namely, that in comparison to induction with $\mathrm{CM}$ physically loaded inside $\mathrm{Cx}-\mathrm{HA}$, the hPLSC-loaded Cx-HA-CM hydrogel induces better chondrogenic differentiation of hPLSCs under the action of the retained CM.

\section{Conclusion}

In this work, we confirmed that an in situ-forming CxHA-CM hydrogel and hPLSCs have good potential for cartilage tissue engineering. The $\mathrm{CM}$ that is retained inside the $\mathrm{Cx}$-HA hydrogel for a long time synergistically induces the chondrogenic differentiation of hPLSCs. Although the exact mechanism of $\mathrm{CM}$ action was not determined in this work, we confirmed that the action of $\mathrm{CM}$ remaining inside the $\mathrm{Cx}-\mathrm{HA}$ for an extended period on cell surface TGF- $\beta$ receptors can synergistically induce the chondrogenic differentiation of hPLSCs for cartilage tissue engineering. The injectable formulation of the $\mathrm{Cx}$ HA-CM-based hydrogel described in this work provides an opportunity to satisfy the unmet need for (pre)clinical repair of damaged articular cartilage.

\section{Acknowledgements}

This study was supported by a grant from the Basic Science Research Program (2016R1A2B3007448) through the National Research Foundation of Korea (NRF) funded by the Ministry of Education and by a grant from the Korea Health Technology R\&D Project (HI17C2191) through the Korea Health Industry Development Institute funded by the Ministry of Health \& Welfare.

\section{Author contributions}

S.H.P., J.Y.S., J.Y.P., Y.B.J., K.K., and J.H.Y. performed the experiments on hyaluronic acid hydrogel preparation, cell viability and CM release. H.S.C. prepared and discussed ZW800-1C. S.H.P., J.Y.S., and Y.B.J. conducted the animal experiments. S.H.P., J.Y.P, K.K., J.H.K., S.C., and M.S.K. prepared and discussed the stained images. M.S.K. designed all of the experiments and wrote the paper. All of the authors discussed the results and commented on the manuscript.

\section{Conflict of interest}

The authors declare that they have no conflict of interest.

\section{Publisher's note}

Springer Nature remains neutral with regard to jurisdictional claims in published maps and institutional affiliations.

Supplementary information is available for this paper at https://doi.org/ 10.1038/s41427-019-0130-1.

Received: 17 January 2019 Revised: 26 March 2019 Accepted: 31 March 2019.

Published online: 21 June 2019

\section{References}

1. Vilela, C. A. et al. Cartilage repair using hydrogels: a critical review of in vivo experimental designs. ACS Biomater. Sci. Eng. 1, 726-739 (2015).

2. Szychlinska, M. A. et al. Mesenchymal stem cell-based cartilageregeneration approach and cell senescence: Can we manipulate cell aging and function? Tissue Eng. Part B Rev. 23, 529-539 (2017).

3. Chu, C. R., Szczodry, M. \& Bruno, S. Animal models for cartilage regeneration and repair. Tissue Eng. Part B Rev. 16, 105-115 (2010).

4. Feng, Q. et al. One-pot solvent exchange preparation of non-swellable, thermoplastic, stretchable and adhesive supramolecular hydrogels based on dual synergistic physical crosslinking. NPG Asia Mat. 10, e455 (2018). 
5. Makris, E. A., Gomoll, A. H., Malizos, K. N., Hu, J. C. \& Athanasiou, K. A. Repair and tissue engineering techniques for articular cartilage. Nat. Rev. Rheuma. 11, 21-34 (2015)

6. Wang, Z. et al. Novel biomaterial strategies for controlled growth factor delivery for biomedical applications. NPG Asia Mat. 9, e435 (2017).

7. Chou, C. L. et al. Micrometer scale guidance of mesenchymal stem cells to form structurally oriented large-scale tissue engineered cartilage. Acta Biomater. 60, 210-219 (2017).

8. Irawan, V., Sung, T., Higuchi, A. \& Ikoma, T. Collagen scaffolds in cartilage tissue engineering and relevant approaches for future development. Tissue Eng. Reg. Med 15, 673-697 (2018).

9. Wang, J., Zhang, F., Tsang, W. P., Wan, C. \& Wu, C. Fabrication of injectable high strength hydrogel based on 4-arm star PEG for cartilage tissue engineering. Biomaterials 120, 11-21 (2017).

10. Gay, I. C., Chen, S. \& MacDougall, M. Isolation and characterization of multipotent human periodontal ligament stem cells. Orthod. Craniofacial Res 10, 149-160 (2007).

11. Charles Huang, C. Y., Pelaez, D., Bendala, J. D., Garcia-Godoy, F. \& Cheung, H. S Plasticity of stem cells derived from adult periodontal ligament. Regen. Med. 4, 809-821 (2009).

12. Um, S. et al. Valproic Acid Modulates the multipotency in periodontal ligament stem cells via p53-mediated cell cycle. Tissue Eng. Reg. Med. 14 153-162 (2017).

13. Collins, M. N. \& Birkinshaw, C. Hyaluronic acid based scaffolds for tissue engineering-A review. Carbohydr. Polym. 92, 1262-1279 (2013).

14. Liang, X., Fang, L., Li, X., Zhang, X. \& Wang, F. Activatable near infrared dye conjugated hyaluronic acid based nanoparticles as a targeted theranostic agent for enhanced fluorescence/CT/photoacoustic imaging guided photothermal therapy. Biomaterials 132, 72-84 (2017).

15. Kim, S. H., In, I. \& Park, S. Y. pH-Responsive NIR-absorbing fluorescent polydopamine with hyaluronic acid for dual targeting and synergistic effects of photothermal and chemotherapy. Biomacromolecules 18, 1825-1835 (2017).

16. Hwang, D. W. et al. In vivo visualization of endogenous miR-21 using hyaluronic acid-coated graphene oxide for targeted cancer therapy. Biomaterials 121, 144-154 (2017).

17. Raia, N. R. et al. Enzymatically crosslinked silk-hyaluronic acid hydrogels. Biomaterials 131, 58-67 (2017).

18. Qu, X., Qiu, P., Zhu, Y., Yang, M. \& Mao, C. Guiding nanomaterials to tumors for breast cancer precision medicine: from tumor-targeting small-molecule discovery to targeted nanodrug delivery. NPG Asia Mat. 9, e452 (2017).

19. Unterman, S. A. et al. Hyaluronic acid-binding scaffold for articular cartilage repair. Tissue Eng. Part A 18, 2497-2506 (2012).

20. Kim, I. L., Khetan, S., Baker, B. M., Chen, C. S. \& Burdick, J. A. Fibrous hyaluronic acid hydrogels that direct MSC chondrogenesis through mechanical and adhesive cues. Biomaterials 34, 5571-5580 (2013).

21. Zhu, D., Wang, H., Trinh, P., Heilshorn, S. C. \& Yang, F. Elastin-like proteinhyaluronic acid (ELP-HA) hydrogels with decoupled mechanical and biochemical cues for cartilage regeneration. Biomaterials 127, 132-140 (2017).

22. Yang, J., Zhang, Y. S., Yue, K. \& Khademhosseini, A. Cell-laden hydrogels for osteochondral and cartilage tissue engineering. Acta Biomater. 57, 1-25 (2017).

23. $Y u, F$. et al. An injectable hyaluronic acid/PEG hydrogel for cartilage tissue engineering formed by integrating enzymatic crosslinking and Diels-Alder "click chemistry". Polym. Chem. 5, 1082-1090 (2014).

24. Liu, J. et al. Pre-vascularization in fibrin Gel/PLGA microsphere scaffolds designed for bone regeneration. NPG Asia Mat. 10, 827-839 (2018).

25. Ren, K., He, C., Xiao, C., Li, G. \& Chen, X. Injectable glycopolypeptide hydrogels as biomimetic scaffolds for cartilage tissue engineering. Biomaterials $\mathbf{5 1}$ 238-249 (2015)
26. Toh, W. S., Lim, T. C., Kurisawa, M. \& Spector, M. Modulation of mesenchymal stem cell chondrogenesis in a tunable hyaluronic acid hydrogel microenvironment. Biomaterials 33, 3835-3845 (2012).

27. Yoon, I. S. et al. Proliferation and chondrogenic differentiation of human adipose-derived mesenchymal stem cells in porous hyaluronic acid scaffold. J. Biosci. Bioeng. 112, 402-408 (2011).

28. Devaraj, N. K., Upadhyay, R., Haun, J. B., Hilderbrand, S. A. \& Weissleder, R. Fast and sensitive pretargeted labeling of cancer cells through a tetrazine/transcyclooctene cycloaddition. Angew. Chem. Int. Ed. Engl. 48, 7013-7016 (2009).

29. Li, Z. et al. Tetrazine-trans-cyclooctene ligation for the rapid construction of ${ }^{18} \mathrm{~F}$ labeled probes. Chem. Commun. 46, 8043-8045 (2010).

30. Liu, D. S. et al. Diels-Alder cycloaddition for fluorophore targeting to specific proteins inside living cells. J. Am. Chem. Soc. 134, 792-795 (2012).

31. Gopinathan, J. \& Noh, I. Click Chemistry-based injectable hydrogels and bioprinting inks for tissue engineering applications. Tissue Eng. Reg. Med $\mathbf{1 5}$ 531-546 (2018)

32. Owen, S. C., Fisher, S. A., Tam, R. Y., Nimmo, C. M. \& Shoichet, M. S. Hyaluronic acid click hydrogels emulate the extracellular matrix. Langmuir 29, 7393-7400 (2013).

33. Weiss, A. \& Attisano, L. The TGFbeta superfamily signaling pathway. Wiley Interdiscip. Rev. Dev. Biol. 2, 47-63 (2013).

34. Lam, H. J., Li, S., Lou, N., Chu, J. \& Bhatnagar, R. S. Synthetic peptides cytomodulin-1 (CM-1) and cytomodulin-2 (CM-2) promote collagen synthesis and wound healing in vitro. Conf. Proc. IEEE Eng. Med. Biol. Soc 7, 5028-5030 (2004).

35. Renner, J. N. \& Liu, J. C. Investigating the effect of peptide agonists on the chondrogenic differentiation of human mesenchymal stem cells using design of experiments. Biotechnol. Prog. 29, 1550-1557 (2013).

36. O'Kane, S. \& Ferguson, M. W. J. Transforming growth factors-b and wound healing. Int. J. Biochem. Cell Biol. 29, 63-78 (1997).

37. Mittal, A., Kumar, R., Parsad, D. \& Kumar, N. Cytomodulin-functionalized porous PLGA particulate scaffolds respond better to cell migration, actin production and wound healing in rodent model. J. Tissue Eng. Regen. Med. 8, 351-363 (2014).

38. Park, S. H. et al. BMP2-modified injectable hydrogel for osteogenic differentiation of human periodontal ligament stem cells. Sci. Rep. 7, 6603 (2017).

39. Hyun, $\mathrm{H}$. et al. Central $\mathrm{C}-\mathrm{C}$ bonding increases optical and chemical stability of NIR fluorophores. RSC Adv. 4, 58762-58768 (2014).

40. Cohen, D. L. et al. Increased mixing improves hydrogel homogeneity and quality of three-dimensional printed constructs. Tissue Eng. Part C. 17, 240-248 (2011).

41. Wang, L. L. et al. Three-dimensional extrusion bioprinting of single- and double-network hydrogels containing dynamic covalent crosslinks. J. Biomed. Mater. Res. A 106, 865-887 (2018).

42. Setzu, A. et al. Inflammation stimulates myelination by transplanted oligodendrocyte precursor cells. Glia 54, 297-303 (2006).

43. Monibi, F. A. \& Cook, J. L. Tissue-derived extracellular matrix bioscaffolds: emerging applications in cartilage and meniscus repair. Tissue Eng. Part B Rev. 23. 386-398 (2017).

44. Lin, Y. et al. Molecular and cellular characterization during chondrogenic differentiation of adipose tissue-derived stromal cells in vitro and cartilage formation in vivo. J. Cell. Mol. Med. 9, 929-939 (2005).

45. Kawaguchi, J., Mee, P. J. \& Smith, A. G. Osteogenic and chondrogenic differentiation of embryonic stem cells in response to specific growth factors. Bone 36, 758-769 (2005).

46. Kronenberg, H. M. Developmental regulation of the growth plate. Nature $\mathbf{4 2 3}$ 332-336 (2003) 